\title{
APPROXIMATION OF EIGENVALUES OF EVOLUTION OPERATORS FOR LINEAR RENEWAL EQUATIONS*
}

\author{
DIMITRI BREDA ${ }^{\dagger}$ AND DAVIDE LIESSI ${ }^{\dagger}$
}

\begin{abstract}
A numerical method based on pseudospectral collocation is proposed to approximate the eigenvalues of evolution operators for linear renewal equations, which are retarded functional equations of Volterra type. Rigorous error and convergence analyses are provided, together with numerical tests. The outcome is an efficient and reliable tool which can be used, for instance, to study the local asymptotic stability of equilibria and periodic solutions of nonlinear autonomous renewal equations. Fundamental applications can be found in population dynamics, where renewal equations play a central role.
\end{abstract}

Key words. renewal equations, Volterra integral equations, retarded functional equations, evolution operators, eigenvalue approximation, pseudospectral collocation, stability, equilibria, periodic solutions

AMS subject classifications. 45C05, 45D05, 47D99, 65L07, 65L15, 65R20

1. Introduction. Delay equations of renewal or differential type are often used in different fields of science to model complex phenomena in a more realistic way, thanks to the presence of delayed terms which relate the current evolution to the past history. Examples of broad areas where delays arise naturally are control theory in engineering $[37,39,53,59]$ and population dynamics or epidemics in mathematical biology [36, 41, 47, 51, 52, 58].

In many applications there is a strong interest in determining the asymptotic stability of particular invariants of the associated dynamical systems, mainly equilibria and periodic solutions. Notable instances are network consensus, mechanical vibrations, endemic states and seasonal fluctuations. The problem is nontrivial since the introduction of delays notoriously requires an infinite-dimensional state space [24].

A common tool to investigate local stability is the principle of linearized stability which, generically, links the stability of a solution of a nonlinear system to that of the null solution of the system linearized around the chosen solution. This linearized system is autonomous in the case of equilibria and has periodic coefficients in the case of periodic solutions.

As far as renewal equations (REs) and retarded functional differential equations (RFDEs) are concerned, the stability of the null solution of a linear autonomous system is determined by the spectrum of the semigroup of solution operators or, equivalently, by that of its infinitesimal generator [25, 31, 40].

For RFDEs, as for ordinary differential equations, the Floquet theory relates the stability of the null solution of a linear periodic system to the characteristic multipliers. These are the eigenvalues of the monodromy operator, i.e., the evolution operator that shifts the state along the solution by one period (see [31, chapter XIV] and $[40$, chapter 8]). An analogous formal theory lacks for REs. A possible extension is still an ongoing effort of the authors and colleagues, in view of the application

*Submitted to the editors July 25, 2017.

Funding: This work was supported by the INdAM GNCS projects "Analisi numerica di certi tipi non classici di equazioni di evoluzione" (2016) and "Analisi e sviluppo di metodologie numeriche per certi tipi non classici di sistemi dinamici" (2017) and the Ph.D. Course in Computer Science, Mathematics and Physics of the University of Udine.

${ }^{\dagger}$ Department of Mathematics, Computer Science and Physics, University of Udine, I-33100 Udine, Italy (dimitri.breda@uniud.it, liessi.davide@spes.uniud.it). 
of sun-star calculus to REs in [25] for equilibria. A preliminary study reveals the above a promising approach, with difficulties restricted to the validation of technical hypotheses. Thus we retain reasonable to assume here the validity of a Floquet theory, as well as that of a corresponding principle of linearized stability (more on this is postponed to section 6).

Given the infinite-dimensional nature of delay equations, numerical methods to approximate the spectrum of the operators mentioned above characterize part of the recent literature (to start see [14] and the references therein). They are based on the reduction to finite dimension, in order to exploit the eigenvalues of the obtained matrices as approximations to (part of) the exact ones.

About equilibria of RFDEs, see [12] for the discretization of the infinitesimal generator via pseudospectral collocation and [34] for the discretization of the solution operator via linear multistep methods. For equilibria of REs and coupled systems of REs and RFDEs, see instead the more recent collocation techniques of $[10,11]$.

Concerning periodic solutions of RFDEs, perhaps the most (indirectly) used technique is that behind DDE-BIFTOOL $[1,57]$, the widespread bifurcation package for delay problems (namely delay differential algebraic equations with constant or state-dependent discrete delays). There, a discretization of the monodromy operator is obtained as a byproduct of the piecewise collocation used to compute periodic solutions [33]. Other approaches are the semi-discretization method [43] and the Chebyshev-based collocations [19, 20, 21], and [44] contains an interesting account of this piece of literature. The most general collocation approach is perhaps [13], targeted to the discretization of generic evolution operators, including both solution operators (for equilibria) and monodromy operators (for periodic solutions, with any ratio between delay and period, even irrational) and any (finite) combination of discrete and distributed delay terms.

From an overall glimpse of the existing works, it emerges clearly that there are no currently available methods to approximate the spectrum of evolution operators of REs. Given their importance in population dynamics [7, 17, 28, 29, 30, 41, 42, 45, $48,52,61$, this lack of tools deserves consideration, especially when the interest is in the stability of periodic solutions. Indeed, inspired by the ideas of the pseudospectral collocation approach for RFDEs of [13], the present work is a first attempt to fill this gap. With respect to [13], in reformulating the evolution operators we introduce an essential modification, in order to accommodate for the different kind of equations. Namely, RFDEs provide the value of the derivative of the unknown function, while REs provide directly the value of the unknown function. Moreover, the state space is a space of $L^{1}$ functions, instead of continuous functions as in the RFDE case; this is a natural choice for REs [25], since in general the initial functions can be discontinuous and the solution itself can be discontinuous at the initial time. Finally, provided that some hypotheses on the integration kernel are satisfied, the right-hand side of REs exhibits a regularizing effect (in the sense that applying the right-hand side to an $L^{1}$ function produces a continuous function), which is not present in general in RFDEs. These differences motivate a complete revisit of [13] rather than a mere adaptation.

A preliminary algorithm implementing the method we propose is adopted for the first time in the recent work [9] for a special class of REs. There it is just marginally summarized, as it is only used in the background simply to support the analysis of the approach for nonlinear problems described in [8]. In this work, instead, the method is central, and we elaborate a full treatment including a rigorous error analysis and 
proof of convergence, as well as numerical tests for experimental confirmation and relevant codes.

The main practical outcome is the construction of an approximating matrix whose eigenvalues are demonstrated to converge to the exact ones, possibly with infinite order, under reasonable regularity assumptions on the model coefficients. This infinite order of convergence, typical of pseudospectral methods [60], represents a key computational feature, especially in case of robust analyses (as for, e.g., stability charts and bifurcations). Indeed, a good accuracy is ensured in general with low matrix dimension and, consequently, low computational cost and time.

For completeness, let us notice that the literature on Volterra integral and functional equations abounds of numerical methods for initial and boundary value problems. The monograph [16] and the references therein may serve as a starting point. However, all these methods deal with time integration to approximate a solution rather than with spectral approximation to detect stability.

The paper is structured as follows. In section 2 we define the problem and reformulate the evolution operators, an essential step hereinafter. In section 3 we define the discretizations of the relevant function spaces and of the generic evolution operator. In section 4 we prove that the discretized evolution operator is well-defined and that its eigenvalues approximate those of the infinite-dimensional evolution operator. In section 5 we present two numerical tests. Concluding comments follow in section 6. Eventually, a matrix representation of the discretized evolution operator is constructed in Appendix A for the sake of implementation and relevant MATLAB codes are available at the authors.

2. Formulation of the problem. For $d \in \mathbb{N}$ and $\tau \in \mathbb{R}$ both positive, consider the function space $X:=L^{1}\left([-\tau, 0], \mathbb{R}^{d}\right)$ equipped with the usual $L^{1}$ norm, denoted by $\|\cdot\|_{X}$. For $s \in \mathbb{R}$ and a function $x$ defined on $[s-\tau,+\infty)$ let

$$
x_{t}(\theta):=x(t+\theta), \quad t \geq s, \theta \in[-\tau, 0] .
$$

Given a measurable function $C:[s,+\infty) \times[-\tau, 0] \rightarrow \mathbb{R}^{d \times d}$ and $\varphi \in X$, define the initial value problem for the $\mathrm{RE}$

$$
x(t)=\int_{-\tau}^{0} C(t, \theta) x_{t}(\theta) \mathrm{d} \theta, \quad t>s,
$$

by imposing $x_{s}=\varphi$. As long as $t \in[0, \tau]$, this corresponds to the Volterra integral equation (VIE) of the second kind

$$
x(t)=\int_{0}^{t} K(t, \sigma) x(\sigma) \mathrm{d} \sigma+f(t)
$$

for

$$
K(t, \sigma):=C(s+t, \sigma-t)
$$

and $f(t):=\int_{t-\tau}^{0} K(t, \sigma) \varphi(\sigma) \mathrm{d} \sigma$. With standard regularity assumptions on the kernel $C$, the solution exists unique and bounded in $L^{1}$ (see Theorem 2.2 below). Moreover, a reasoning on the lines of Bellman's method of steps $[3,5]$ allows to extend wellposedness to any $t>s$, by working successively on $[\tau, 2 \tau],[2 \tau, 3 \tau]$ and so on (see also 
$[2,4]$ for similar arguments, and [16, section 4.1.2] for VIEs). Denote this solution by $x(t)$, or $x(t ; s, \varphi)$ when emphasis on $s$ and $\varphi$ is required.

Let $\{T(t, s)\}_{t \geq s}$ be the family of linear and bounded evolution operators [23, 31] associated to (2.2), i.e.,

$$
T(t, s): X \rightarrow X, \quad T(t, s) \varphi=x_{t}(\cdot ; s, \varphi) .
$$

The aim of this work is to approximate the dominant part of the spectrum of the infinite-dimensional operator $T(t, s)$ for the sake of studying stability. This is pursued by reducing to finite dimension via the pseudospectral collocation described in section 3 and by using the eigenvalues of the obtained matrix, computed via standard techniques, as approximations to the exact ones.

Let, e.g., $C(t, \theta)$ be $\Omega$-periodic in $t$. As anticipated in section 1, we assume the validity of a Floquet theory and of a corresponding principle of linearized stability. Thus, the eigenvalues of the monodromy operator $T(\Omega, 0)$, called characteristic multipliers, provide information on the stability of the null solution of (2.2). Moreover, if (2.2) comes from the linearization of a nonlinear RE around a periodic solution, the multipliers reveal also the local stability of the latter. More precisely, except for the trivial multiplier 1, which is always present due to linearization but does not affect stability, the original periodic solution is locally asymptotically stable if all the multipliers are inside the unit circle. Otherwise, a multiplier outside the unit circle is enough to declare instability.

The same reasoning can be applied equally to $T(h, 0)$, independently of $h>0$, to study the stability of the null solution of (2.2) in the autonomous case, i.e., when $C(t, \theta)$ is independent of $t$. By linearization, again, this is valid also for equilibria of nonlinear systems. Here the evolution family reduces to a classic one-parameter semigroup, whose generator can be discretized as in [10] or [11], as already mentioned, providing alternatives to the method described in this work.

One can use the discretization we propose in the framework of [15] also to compute Lyapunov exponents for the generic nonautonomous case. Preliminary results appear already in [9] and are confirmed by the ones obtained therein for equilibria and periodic solutions, with reference to negative and zero exponents, respectively. For further comments on this topic see section 6 .

To keep this level of generality, embracing autonomous, periodic and generic nonautonomous problems altogether, let $h \in \mathbb{R}$ be positive and define for brevity

$$
T:=T(s+h, s) .
$$

From now on this is the generic evolution operator that we aim at discretizing. We remark that any relation between $h$ and $\tau$, even irrational, is allowed.

The following reformulation of $T$ is inspired by the one used in [13] for RFDEs. It is convenient for discretizing $T$ and approximating its eigenvalues. With respect to [13], an essential modification of the operator $V$ below is introduced to take into account the different way by which the equation describes the solution, i.e., directly (REs) or through its derivative (RFDEs).

Define the function spaces $X^{+}:=L^{1}\left([0, h], \mathbb{R}^{d}\right)$ and $X^{ \pm}:=L^{1}\left([-\tau, h], \mathbb{R}^{d}\right)$, equipped with the corresponding $L^{1}$ norms denoted, respectively, by $\|\cdot\|_{X^{+}}$and $\|\cdot\|_{X^{ \pm}}$. 
Define the operator $V: X \times X^{+} \rightarrow X^{ \pm}$as

$$
V(\varphi, w)(t):= \begin{cases}w(t), & t \in(0, h] \\ \varphi(t), & t \in[-\tau, 0]\end{cases}
$$

Let also $V^{-}: X \rightarrow X^{ \pm}$and $V^{+}: X^{+} \rightarrow X^{ \pm}$be given, respectively, by $V^{-} \varphi:=$ $V\left(\varphi, 0_{X^{+}}\right)$and $V^{+} w:=V\left(0_{X}, w\right)$, where $0_{Y}$ denotes the null element of a linear space $Y$ (similarly, $I_{Y}$ in the sequel stands for the identity operator in $Y$ ). Observe that

$$
V(\varphi, w)=V^{-} \varphi+V^{+} w .
$$

Note as much that $V(\varphi, w)$ can have a discontinuity in 0 even when $\varphi$ and $w$ are continuous but $\varphi(0) \neq w(0)$. This is an important difference with respect to [13], which calls later on for special attention to discontinuities and to the role of 0 , both in the theoretical treatment of the numerical method and in its implementation.

Remark 2.1. The choice of including $t=0$ in the past in (2.5), as well as in (2.2), is common for REs modeling, e.g., structured populations [25, 27]. From the theoretical point of view, it does not make any difference, since $X$ consists of equivalence classes of functions coinciding almost everywhere. From the interpretative point of view, it can be motivated by the consideration that although the actual value $\varphi(0)$ is not well-defined, being $\varphi$ in $L^{1}$, it is reasonable to define the solution as coinciding with the initial function $\varphi$ of the problem on the whole domain of $\varphi$. Moreover, from the implementation point of view, numerical tests performed including $t=0$ in the past or in the future show that either choice gives the same results, with the only (obvious) requirement to be consistent throughout the code.

Now define also the operator $\mathcal{F}_{s}: X^{ \pm} \rightarrow X^{+}$as

$$
\mathcal{F}_{s} u(t):=\int_{-\tau}^{0} C(s+t, \theta) u(t+\theta) \mathrm{d} \theta, \quad t \in[0, h] .
$$

Eventually, the evolution operator $T$ can be reformulated as

$$
T \varphi=V\left(\varphi, w^{*}\right)_{h},
$$

where $w^{*} \in X^{+}$is the solution of the fixed point equation

$$
w=\mathcal{F}_{s} V(\varphi, w),
$$

which exists unique and bounded thanks to Theorem 2.2 below (where in (2.10), and also in the sequel, $|\cdot|$ denotes any finite-dimensional norm). Recall that in (2.8) the subscript $h$ is used according to $(2.1)$, hence $V\left(\varphi, w^{*}\right)_{h}(\theta)=V\left(\varphi, w^{*}\right)(h+\theta)$ for $\theta \in[-\tau, 0]$.

THEOREM 2.2. If the interval $[0, \tau]$ can be partitioned into finitely many subintervals $J_{1}, \ldots, J_{n}$ such that, for any $s \in \mathbb{R}$,

$$
\underset{\sigma \in J_{i}}{\operatorname{ess} \sup _{J_{i}}} \int_{J_{i}}|C(s+t, \sigma-t)| \mathrm{d} t<1, \quad i \in\{1, \ldots, n\},
$$

then the operator $I_{X^{+}}-\mathcal{F}_{s} V^{+}$is invertible with bounded inverse and (2.9) admits a unique solution in $X^{+}$. 
Proof. Given $f \in X^{+}$the equation $\left(I_{X^{+}}-\mathcal{F}_{s} V^{+}\right) w=f$ has a unique solution $w \in X^{+}$if and only if the initial value problem

$$
\left\{\begin{array}{l}
w(t)=\int_{-\tau}^{0} C(s+t, \theta) w(t+\theta) \mathrm{d} \theta+f(t), \quad t \in[0, h], \\
w_{0}=0 \in X
\end{array}\right.
$$

has a unique solution in $X^{ \pm}$, with the two solutions coinciding on $[0, h]$. If $h \leq \tau$, this follows directly from standard theory on VIEs, see, e.g., [38, Corollary 9.3.14 and Theorem 9.3.6], whose validity is ensured via (2.3) by the hypothesis on $C$. Otherwise, the same argument can be repeated on $[\tau, 2 \tau],[2 \tau, 3 \tau]$ and so on. So $I_{X^{+}}-\mathcal{F}_{s} V^{+}$is invertible and bounded and the bounded inverse theorem completes the proof.

We conclude this section by comparing the choice of (2.2) as a prototype equation to that of the general linear nonautonomous RFDE [13, (2.1)] (or, equivalently, [14, $(2.4)])$, i.e., $x^{\prime}(t)=L(t) x_{t}$ for linear bounded operators $L(t): X \rightarrow \mathbb{R}^{d}, t \geq s$. Thanks to the Riesz representation theorem for $L^{1}$ (see, e.g., [56, page 400]), every linear nonautonomous retarded functional equation of the type $x(t)=L(t) x_{t}$ can be written in the form (2.2), although not all of them satisfy the assumptions of Theorem 2.2. Think, e.g., of the difference equation $x(t)=a(t) x(t-\tau)$, i.e., $C(t, \theta)=a(t) \delta_{-\tau}(\theta)$ for $\delta_{-\tau}$ the Dirac delta at $-\tau$. Here we exclude these equations because, first and as already noted, they might not be well-posed. Second, they do not ensure the regularization of solutions as it happens for the analogous RFDEs, and this is fundamental for the convergence of the numerical method. Third and last, they might be of neutral type, a case out of the scope of the present work and about which we comment further in section 6.

Also with reference to $[13,(2.4)]$, in many applications the function $C(t, \theta)$ (is continuous in $t$ and) has a finite number of discontinuities in $\theta$. Hence (2.2) may often be written in the form

$$
x(t)=\sum_{k=1}^{p} \int_{-\tau_{k}}^{-\tau_{k-1}} C_{k}(t, \theta) x(t+\theta) \mathrm{d} \theta
$$

with $\tau_{0}:=0<\tau_{1}<\cdots<\tau_{p}:=\tau$ and $C_{k}(t, \theta)$ continuous in $\theta$. In section 5 we refer to this choice, which agrees, for instance, with the literature on physiologically- and age-structured populations (where discontinuities are due, e.g., to different behavior of juveniles and adults) [29, 41, 52].

3. Discretization. In order to approximate the eigenvalues of the infinite-dimensional operator $T: X \rightarrow X$ defined in (2.4), we discretize the function spaces and the operator itself by revisiting the pseudospectral collocation method used in [13], with the necessary modifications due to the new definition of $V$ and those anticipated in section 1.

In the sequel let $M$ and $N$ be positive integers, referred to as discretization indices.

3.1. Partition of time intervals. If $h \geq \tau$, let $\Omega_{M}:=\left\{\theta_{M, 0}, \ldots, \theta_{M, M}\right\}$ be a partition of $[-\tau, 0]$ with $-\tau=\theta_{M, M}<\cdots<\theta_{M, 0}=0$. If $h<\tau$, instead, let $Q$ be the minimum positive integer $q$ such that $q h \geq \tau$. Note that $Q>1$. Let $\theta^{(q)}:=-q h$ for $q \in\{0, \ldots, Q-1\}$ and $\theta^{(Q)}:=-\tau$. For $q \in\{1, \ldots, Q\}$, let $\Omega_{M}^{(q)}:=\left\{\theta_{M, 0}^{(q)}, \ldots, \theta_{M, M}^{(q)}\right\}$ 
be a partition of $\left[\theta^{(q)}, \theta^{(q-1)}\right]$ with

$$
\begin{aligned}
\theta^{(1)} & =\theta_{M, M}^{(1)}<\cdots<\theta_{M, 0}^{(1)}=\theta^{(0)}=0, \\
\theta^{(q)} & =\theta_{M, M}^{(q)}<\cdots<\theta_{M, 0}^{(q)}=\theta^{(q-1)}, \quad q \in\{2, \ldots, Q-1\}, \\
-\tau=\theta^{(Q)} & =\theta_{M, M}^{(Q)}<\cdots<\theta_{M, 0}^{(Q)}=\theta^{(Q-1)} .
\end{aligned}
$$

Define also the partition $\Omega_{M}:=\Omega_{M}^{(1)} \cup \cdots \cup \Omega_{M}^{(Q)}$ of $[-\tau, 0]$. Note in particular that for $q \in\{1, \ldots, Q-1\}$

$$
\theta_{M, M}^{(q)}=-q h=\theta_{M, 0}^{(q+1)} .
$$

In principle, one can use more general meshes in $[-\tau, 0]$, e.g., not including the endpoints or using different families of nodes in the piecewise case. The forthcoming results can be generalized straightforwardly, but we avoid this choice in favor of a lighter notation and to reduce technicalities.

Finally, let $\Omega_{N}^{+}:=\left\{t_{N, 1}, \ldots, t_{N, N}\right\}$ be a partition of $[0, h]$ with $0 \leq t_{N, 1}<\cdots<$ $t_{N, N} \leq h$.

3.2. Discretization of function spaces. If $h \geq \tau$, the discretization of $X$ of index $M$ is $X_{M}:=\mathbb{R}^{d(M+1)}$. An element $\Phi \in X_{M}$ is written as $\Phi=\left(\Phi_{0}, \ldots, \Phi_{M}\right)$, where $\Phi_{m} \in \mathbb{R}^{d}$ for $m \in\{0, \ldots, M\}$. The restriction operator $R_{M}: \tilde{X} \rightarrow X_{M}$ is given by $R_{M} \varphi:=\left(\varphi\left(\theta_{M, 0}\right), \ldots, \varphi\left(\theta_{M, M}\right)\right)$ for $\tilde{X}$ any subspace of $X$ regular enough to make point-wise evaluation meaningful. The same holds below and see also the comment concluding this section. The prolongation operator $P_{M}: X_{M} \rightarrow X$ is the discrete Lagrange interpolation operator $P_{M} \Phi(\theta):=\sum_{m=0}^{M} \ell_{M, m}(\theta) \Phi_{m}, \theta \in[-\tau, 0]$, where $\ell_{M, 0}, \ldots, \ell_{M, M}$ are the Lagrange coefficients relevant to the nodes of $\Omega_{M}$. Observe that

$$
R_{M} P_{M}=I_{X_{M}}, \quad P_{M} R_{M}=\mathcal{L}_{M},
$$

where $\mathcal{L}_{M}: \tilde{X} \rightarrow X$ is the Lagrange interpolation operator that associates to a function $\varphi \in \tilde{X}$ the $M$-degree $\mathbb{R}^{d}$-valued polynomial $\mathcal{L}_{M} \varphi$ such that $\mathcal{L}_{M} \varphi\left(\theta_{M, m}\right)=$ $\varphi\left(\theta_{M, m}\right)$ for $m \in\{0, \ldots, M\}$.

If $h<\tau$, proceed similarly but in a piecewise fashion. The discretization of $X$ of index $M$ is $X_{M}:=\mathbb{R}^{d(Q M+1)}$. An element $\Phi \in X_{M}$ is written as

$$
\Phi=\left(\Phi_{0}^{(1)}, \ldots, \Phi_{M-1}^{(1)}, \ldots, \Phi_{0}^{(Q)}, \ldots, \Phi_{M-1}^{(Q)}, \Phi_{M}^{(Q)}\right),
$$

where $\Phi_{m}^{(q)} \in \mathbb{R}^{d}$ for $q \in\{1, \ldots, Q\}$ and $m \in\{0, \ldots, M-1\}$ and $\Phi_{M}^{(Q)} \in \mathbb{R}^{d}$. In view of (3.1), let also $\Phi_{M}^{(q)}:=\Phi_{0}^{(q+1)}$ for $q \in\{1, \ldots, Q-1\}$. The restriction operator $R_{M}: \tilde{X} \rightarrow X_{M}$ is given by

$$
R_{M} \varphi:=\left(\varphi\left(\theta_{M, 0}^{(1)}\right), \ldots, \varphi\left(\theta_{M, M-1}^{(1)}\right), \ldots, \varphi\left(\theta_{M, 0}^{(Q)}\right), \ldots, \varphi\left(\theta_{M, M-1}^{(Q)}\right), \varphi\left(\theta_{M, M}^{(Q)}\right)\right) .
$$

The prolongation operator $P_{M}: X_{M} \rightarrow X$ is the discrete piecewise Lagrange interpolation operator $P_{M} \Phi(\theta):=\sum_{m=0}^{M} \ell_{M, m}^{(q)}(\theta) \Phi_{m}^{(q)}, \theta \in\left[\theta^{(q)}, \theta^{(q-1)}\right], q \in\{1, \ldots, Q\}$, where $\ell_{M, 0}^{(q)}, \ldots, \ell_{M, M}^{(q)}$ are the Lagrange coefficients relevant to the nodes of $\Omega_{M}^{(q)}$ for

\footnotetext{
$\ddagger$ Throughout the text we use this simpler notation to denote a concatenation of column vectors in place of the more formal $\Phi=\left(\Phi_{0}^{T}, \ldots, \Phi_{M}^{T}\right)^{T}$.
} 
$q \in\{1, \ldots, Q\}$. Observe that the equalities (3.2) hold again, with $\mathcal{L}_{M}: \tilde{X} \rightarrow X$ the piecewise Lagrange interpolation operator that associates to a function $\varphi \in \tilde{X}$ the piecewise polynomial $\mathcal{L}_{M} \varphi$ such that $\mathcal{L}_{M} \varphi\left\lceil_{\left[\theta^{(q)}, \theta^{(q-1)}\right]}\right.$ is the $M$-degree $\mathbb{R}^{d}$-valued polynomial with values $\varphi\left(\theta_{M, m}^{(q)}\right)$ at the nodes $\theta_{M, m}^{(q)}$ for $q \in\{1, \ldots, Q\}$ and $m=0, \ldots, M$. Notice that to avoid a cumbersome notation the same symbols for $X_{M}, R_{M}, P_{M}$ and $\mathcal{L}_{M}$ are used.

Finally, the discretization of $X^{+}$of index $N$ is $X_{N}^{+}:=\mathbb{R}^{d N}$. An element $W \in X_{N}^{+}$ is written as $W=\left(W_{1}, \ldots, W_{N}\right)$, where $W_{n} \in \mathbb{R}^{d}$ for $n \in\{1, \ldots, N\}$. The restriction operator $R_{N}^{+}: \tilde{X}^{+} \rightarrow X_{N}^{+}$is given by $R_{N}^{+} w:=\left(w\left(t_{N, 1}\right), \ldots, w\left(t_{N, N}\right)\right)$. The prolongation operator $P_{N}^{+}: X_{N}^{+} \rightarrow X^{+}$is the discrete Lagrange interpolation operator $P_{N}^{+} W(t):=\sum_{n=1}^{N} \ell_{N, n}^{+}(t) W_{n}, t \in[0, h]$, where $\ell_{N, 1}^{+}, \ldots, \ell_{N, N}^{+}$are the Lagrange coefficients relevant to the nodes of $\Omega_{N}^{+}$. Observe again that

$$
R_{N}^{+} P_{N}^{+}=I_{X_{N}^{+}}, \quad P_{N}^{+} R_{N}^{+}=\mathcal{L}_{N}^{+},
$$

where $\mathcal{L}_{N}^{+}: \tilde{X}^{+} \rightarrow X^{+}$is the Lagrange interpolation operator that associates to a function $w \in \tilde{X}^{+}$the $(N-1)$-degree $\mathbb{R}^{d}$-valued polynomial $\mathcal{L}_{N}^{+} w$ such that $\mathcal{L}_{N}^{+} w\left(t_{N, n}\right)=$ $w\left(t_{N, n}\right)$ for $n \in\{1, \ldots, N\}$.

When not ambiguous (e.g., when applied to an element) the restrictions to subspaces of the above prolongation, restriction and Lagrange interpolation operators are denoted in the same way as the operators themselves.

Observe that since an $L^{1}$ function is an equivalence class of functions equal almost everywhere, values in specific points are not well-defined. Thus, it does not seem reasonable to define the restriction operator on the whole space $X$ (respectively, $X^{+}$), motivating the above use of $\tilde{X}$ (respectively, $\tilde{X}^{+}$). Indeed, this is amply justified. First of all, it is clear from the following sections that the restriction and interpolation operators are actually applied only to continuous functions or polynomials (or their piecewise counterparts if $h<\tau)$. Moreover, the interest of the present work is in the eigenfunctions of the evolution operator (see Theorem 4.10 below), which are expected to be sufficiently regular (see relevant comments in section 6). As a last argument, ultimately, the numerical method is applied to finite-dimensional vectors, which bear no notion of the function from which they are derived.

3.3. Discretization of $T$. Following (2.8) and (2.9), the discretization of indices $M$ and $N$ of the evolution operator $T$ in (2.4) is the finite-dimensional operator $T_{M, N}: X_{M} \rightarrow X_{M}$ defined as

$$
T_{M, N} \Phi:=R_{M} V\left(P_{M} \Phi, P_{N}^{+} W^{*}\right)_{h},
$$

where $W^{*} \in X_{N}^{+}$is a solution of the fixed point equation

$$
W=R_{N}^{+} \mathcal{F}_{s} V\left(P_{M} \Phi, P_{N}^{+} W\right)
$$

for the given $\Phi \in X_{M}$. We establish that (3.5) is well-posed in subsection 4.2.

By virtue of (2.6), the operator $T_{M, N}$ can be rewritten as

$$
T_{M, N} \Phi=T_{M}^{(1)} \Phi+T_{M, N}^{(2)} W^{*},
$$

with $T_{M}^{(1)}: X_{M} \rightarrow X_{M}$ and $T_{M, N}^{(2)}: X_{N}^{+} \rightarrow X_{M}$ defined as

$$
T_{M}^{(1)} \Phi:=R_{M}\left(V^{-} P_{M} \Phi\right)_{h}, \quad T_{M, N}^{(2)} W:=R_{M}\left(V^{+} P_{N}^{+} W\right)_{h} .
$$


Similarly, the fixed point equation (3.5) can be rewritten as

$$
\left(I_{X_{N}^{+}}-U_{N}^{(2)}\right) W=U_{M, N}^{(1)} \Phi,
$$

with $U_{M, N}^{(1)}: X_{M} \rightarrow X_{N}^{+}$and $U_{N}^{(2)}: X_{N}^{+} \rightarrow X_{N}^{+}$defined as

$$
U_{M, N}^{(1)} \Phi:=R_{N}^{+} \mathcal{F}_{s} V^{-} P_{M} \Phi, \quad U_{N}^{(2)} W:=R_{N}^{+} \mathcal{F}_{s} V^{+} P_{N}^{+} W .
$$

Since $I_{X_{N}^{+}}-U_{N}^{(2)}$ is invertible, the operator $T_{M, N}: X_{M} \rightarrow X_{M}$ can be eventually reformulated as

$$
T_{M, N}=T_{M}^{(1)}+T_{M, N}^{(2)}\left(I_{X_{N}^{+}}-U_{N}^{(2)}\right)^{-1} U_{M, N}^{(1)} .
$$

This reformulation simplifies the construction of the matrix representation of $T_{M, N}$ given in Appendix A.

4. Convergence analysis. After introducing some additional spaces and assumptions in subsection 4.1, we first prove that the discretized problem (viz. (3.5)) is well-posed in subsection 4.2. Then, in subsection 4.3, we present the proof of the convergence of the eigenvalues of the finite-dimensional operator $T_{M, N}$ to those of the infinite-dimensional operator $T$.

4.1. Additional spaces and assumptions. Consider the space of continuous functions $X_{C}^{+}:=C\left([0, h], \mathbb{R}^{d}\right) \subset X^{+}$equipped with the uniform norm, denoted by $\|\cdot\|_{X_{C}^{+}}$. If $h \geq \tau$ consider also $X_{C}:=C\left([-\tau, 0], \mathbb{R}^{d}\right) \subset X$ equipped with the uniform norm, denoted by $\|\cdot\|_{X_{C}}$. If $h<\tau$, instead, define

$$
\begin{aligned}
X_{C}:=\{\varphi \in X \mid & \varphi_{\left(\theta^{(q+1)}, \theta^{(q)}\right)} \in C\left(\left(\theta^{(q+1)}, \theta^{(q)}\right), \mathbb{R}^{d}\right), q \in\{0, \ldots, Q-1\} \\
& \text { and the one-sided limits at } \left.\theta^{(q)} \text { exist finite, } q \in\{0, \ldots, Q\}\right\} \subset X,
\end{aligned}
$$

equipped with the same norm $\|\cdot\|_{X_{C}}$. With these choices, all these function spaces are Banach spaces.

Remark 4.1. Observe that $X_{C}$ and $X_{C}^{+}$are identified with their projections on the spaces $X$ and $X^{+}$, respectively, hence their elements may be seen as equivalence classes of functions coinciding almost everywhere. In particular, the values of a function in $X$ or $X^{+}$at the endpoints of the domain interval are not relevant to that function being an element of $X_{C}$ or $X_{C}^{+}$, respectively. The same is true for the endpoints of domain pieces for elements of $X_{C}$ if $h<\tau$.

In the following sections, some hypotheses on the discretization nodes in $[0, h]$ and on $\mathcal{F}_{s}$ and $V$ are needed beyond the assumption of Theorem 2.2, in order to attain the regularity required to ensure the convergence of the method. They are all referenced individually from the following list where needed:

(H1) the meshes $\left\{\Omega_{N}^{+}\right\}_{N>0}$ are the Chebyshev zeros

$$
t_{N, n}:=\frac{h}{2}\left(1-\cos \left(\frac{(2 n-1) \pi}{2 N}\right)\right), \quad n \in\{1, \ldots, N\} ;
$$

(H2) the hypothesis of Theorem 2.2 holds; 
(H3) $\mathcal{F}_{s} V^{+}: X^{+} \rightarrow X^{+}$has range contained in $X_{C}^{+}$and $\mathcal{F}_{s} V^{+}: X^{+} \rightarrow X_{C}^{+}$is bounded;

(H4) $\mathcal{F}_{s} V^{-}: X \rightarrow X^{+}$has range contained in $X_{C}^{+}$and $\mathcal{F}_{s} V^{-}: X \rightarrow X_{C}^{+}$is bounded.

With respect to (2.5) and (2.7), hypotheses (H3) and (H4) are fulfilled if the following two conditions on the kernel $C$ of (2.2) are satisfied:

(C1) there exists $\gamma>0$ such that $|C(t, \theta)| \leq \gamma$ for all $t \in[0, h]$ and almost all $\theta \in[-\tau, 0]$

(C2) $t \mapsto C(t, \theta)$ is continuous for almost all $\theta \in[-\tau, 0]$, uniformly with respect to $\theta$.

Indeed, let $u \in X^{ \pm} \backslash\{0\}, t \in[0, h]$ and $\epsilon>0$. From the continuity of translation in $L^{1}$ there exists $\delta^{\prime}>0$ such that for all $t^{\prime} \in[0, h]$ if $\left|t^{\prime}-t\right|<\delta^{\prime}$ then $\int_{-\tau}^{0} \mid u\left(t^{\prime}+\theta\right)-$ $u(t+\theta) \mid \mathrm{d} \theta<\frac{\epsilon}{2 \gamma}$. From condition (C2) there exists $\delta^{\prime \prime}>0$ such that for all $t^{\prime} \in[0, h]$ and almost all $\theta \in[-\tau, 0]$ if $\left|t^{\prime}-t\right|<\delta^{\prime \prime}$ then $\left|C\left(t^{\prime}, \theta\right)-C(t, \theta)\right|<\frac{\epsilon}{2\|u\|_{X^{ \pm}}}$. Hence, for all $t^{\prime} \in[0, h]$ if $\left|t^{\prime}-t\right|<\delta:=\min \left\{\delta^{\prime}, \delta^{\prime \prime}\right\}$ then

$$
\begin{aligned}
& \left|\int_{-\tau}^{0} C\left(t^{\prime}, \theta\right) u\left(t^{\prime}+\theta\right) \mathrm{d} \theta-\int_{-\tau}^{0} C(t, \theta) u(t+\theta) \mathrm{d} \theta\right| \\
& \quad \leq \int_{-\tau}^{0}\left|C\left(t^{\prime}, \theta\right)\right|\left|u\left(t^{\prime}+\theta\right)-u(t+\theta)\right| \mathrm{d} \theta+\int_{-\tau}^{0}\left|C\left(t^{\prime}, \theta\right)-C(t, \theta)\right||u(t+\theta)| \mathrm{d} \theta \\
& \quad<\gamma \frac{\epsilon}{2 \gamma}+\frac{\epsilon}{2\|u\|_{X^{ \pm}}} \int_{-\tau}^{0}|u(t+\theta)| \mathrm{d} \theta \leq \epsilon .
\end{aligned}
$$

Since $\mathcal{F}_{s} 0_{X^{ \pm}}=0_{X^{+}}$, this shows that $\mathcal{F}_{s}\left(X^{ \pm}\right) \subset X_{C}^{+}$, which implies the first part of hypotheses (H3) and (H4). Boundedness follows immediately. Eventually, observe that condition $(\mathrm{C} 1)$ implies also hypothesis $(\mathrm{H} 2)$. Indeed, the interval $[0, \tau]$ can be partitioned into finitely many subintervals $J_{1}, \ldots, J_{n}$, each of length less than $\frac{1}{\gamma}$, such that, for any $s \in \mathbb{R}$ and all $i \in\{1, \ldots, n\}$,

$$
\underset{\sigma \in J_{i}}{\operatorname{ess} \sup } \int_{J_{i}}|C(s+t, \sigma-t)| \mathrm{d} t \leq \gamma \int_{J_{i}} \mathrm{~d} t<1 .
$$

Anyway, in the sequel we base the proofs on hypotheses (H2) to (H4) in the case one uses operators $V$ and $\mathcal{F}_{s}$ more general than or different from (2.5) and (2.7).

4.2. Well-posedness of the collocation equation. With reference to (3.5), let $\varphi \in X$ and consider the collocation equation

$$
W=R_{N}^{+} \mathcal{F}_{s} V\left(\varphi, P_{N}^{+} W\right)
$$

in $W \in X_{N}^{+}$. The aim of this section is to show that (4.1) has a unique solution and to study its relation to the unique solution $w^{*} \in X^{+}$of (2.9). Using (2.6), the equations (2.9) and (4.1) can be rewritten, respectively, as $\left(I_{X^{+}}-\mathcal{F}_{s} V^{+}\right) w=\mathcal{F}_{s} V^{-} \varphi$ and

$$
\left(I_{X_{N}^{+}}-R_{N}^{+} \mathcal{F}_{s} V^{+} P_{N}^{+}\right) W=R_{N}^{+} \mathcal{F}_{s} V^{-} \varphi
$$

The following preliminary result concerns the operators

$$
I_{X^{+}}-\mathcal{L}_{N}^{+} \mathcal{F}_{s} V^{+}: X^{+} \rightarrow X^{+},
$$


and

$$
I_{X_{N}^{+}}-R_{N}^{+} \mathcal{F}_{s} V^{+} P_{N}^{+}: X_{N}^{+} \rightarrow X_{N}^{+} .
$$

Proposition 4.2. If the operator (4.3) is invertible, then the operator (4.4) is invertible. Moreover, given $\bar{W} \in X_{N}^{+}$, the unique solution $\hat{w} \in X^{+}$of

$$
\left(I_{X^{+}}-\mathcal{L}_{N}^{+} \mathcal{F}_{s} V^{+}\right) w=P_{N}^{+} \bar{W}
$$

and the unique solution $\hat{W} \in X_{N}^{+}$of

$$
\left(I_{X_{N}^{+}}-R_{N}^{+} \mathcal{F}_{s} V^{+} P_{N}^{+}\right) W=\bar{W}
$$

are related by $\hat{W}=R_{N}^{+} \hat{w}$ and $\hat{w}=P_{N}^{+} \hat{W}$.

Proof. If (4.3) is invertible, then, given $\bar{W} \in X_{N}^{+}$, (4.5) has a unique solution, say $\hat{w} \in X^{+}$. Then, by (3.4),

$$
\hat{w}=P_{N}^{+}\left(R_{N}^{+} \mathcal{F}_{s} V^{+} \hat{w}+\bar{W}\right)
$$

and

$$
R_{N}^{+} \hat{w}=R_{N}^{+} \mathcal{F}_{s} V^{+} \hat{w}+\bar{W}
$$

hold. Hence, by substituting (4.8) in (4.7),

$$
\hat{w}=P_{N}^{+} R_{N}^{+} \hat{w}
$$

and, by substituting (4.9) in (4.8), $R_{N}^{+} \hat{w}=R_{N}^{+} \mathcal{F}_{s} V^{+} P_{N}^{+} R_{N}^{+} \hat{w}+\bar{W}$, i.e., $R_{N}^{+} \hat{w}$ is a solution of (4.6).

Vice versa, if $\hat{W} \in X_{N}^{+}$is a solution of (4.6), then $P_{N}^{+} \hat{W}=\mathcal{L}_{N}^{+} \mathcal{F}_{s} V^{+} P_{N}^{+} \hat{W}+P_{N}^{+} \bar{W}$ holds again by (3.4), i.e., $P_{N}^{+} \hat{W}$ is a solution of (4.5). Hence, by uniqueness, $\hat{w}=P_{N}^{+} \hat{W}$ holds.

Finally, if $\hat{W}_{1}, \hat{W}_{2} \in X_{N}^{+}$are solutions of (4.6), then $P_{N}^{+} \hat{W}_{1}=\hat{w}=P_{N}^{+} \hat{W}_{2}$ and, once again by (3.4), $\hat{W}_{1}=R_{N}^{+} P_{N}^{+} \hat{W}_{1}=R_{N}^{+} P_{N}^{+} \hat{W}_{2}=\hat{W}_{2}$. Therefore $\hat{W}:=R_{N}^{+} \hat{w}$ is the unique solution of (4.6) and the operator (4.4) is invertible.

As observed above, the equation (4.1) is equivalent to (4.2), hence, by choosing

$$
\bar{W}=R_{N}^{+} \mathcal{F}_{s} V^{-} \varphi,
$$

it is equivalent to (4.6). Observe also that thanks to (3.4) the equation

$$
w=\mathcal{L}_{N}^{+} \mathcal{F}_{s} V(\varphi, w)
$$

can be rewritten as $\left(I_{X^{+}}-\mathcal{L}_{N}^{+} \mathcal{F}_{s} V^{+}\right) w=\mathcal{L}_{N}^{+} \mathcal{F}_{s} V^{-} \varphi=P_{N}^{+} R_{N}^{+} \mathcal{F}_{s} V^{-} \varphi$, which is equivalent to (4.5) with the choice (4.10). Thus, by Proposition 4.2, if the operator (4.3) is invertible, then the equation (4.1) has a unique solution $W^{*} \in X_{N}^{+}$such that

$$
W^{*}=R_{N}^{+} w_{N}^{*}, \quad w_{N}^{*}=P_{N}^{+} W^{*},
$$

where $w_{N}^{*} \in X^{+}$is the unique solution of (4.11). Note for clarity that (4.10) implies $w_{N}^{*}=\hat{w}$ for $\hat{w}$ in Proposition 4.2. So, now we show that (4.3) is invertible under due assumptions. 
Proposition 4.3. If hypotheses (H1) to (H3) hold, then there exists a positive integer $N_{0}$ such that, for any $N \geq N_{0}$, the operator (4.3) is invertible and

$$
\left\|\left(I_{X^{+}}-\mathcal{L}_{N}^{+} \mathcal{F}_{s} V^{+}\right)^{-1}\right\|_{X^{+} \leftarrow X^{+}} \leq 2\left\|\left(I_{X^{+}}-\mathcal{F}_{s} V^{+}\right)^{-1}\right\|_{X^{+} \leftarrow X^{+}} .
$$

Moreover, for each $\varphi \in X$, (4.11) has a unique solution $w_{N}^{*} \in X^{+}$and

$$
\left\|w_{N}^{*}-w^{*}\right\|_{X^{+}} \leq 2\left\|\left(I_{X^{+}}-\mathcal{F}_{s} V^{+}\right)^{-1}\right\|_{X^{+} \leftarrow X^{+}}\left\|\mathcal{L}_{N}^{+} w^{*}-w^{*}\right\|_{X^{+}},
$$

where $w^{*} \in X^{+}$is the unique solution of (2.9).

Proof. In this proof, let $I:=I_{X^{+}}$. By [35, Corollary of Theorem Ia], assuming hypothesis (H1), if $w \in X_{C}^{+}$, then $\left\|\left(\mathcal{L}_{N}^{+}-I\right) w\right\|_{X^{+}} \rightarrow 0$ for $N \rightarrow \infty$. By the BanachSteinhaus theorem, the sequence $\left\|\left.\left(\mathcal{L}_{N}^{+}-I\right)\right|_{X_{C}^{+}}\right\|_{X^{+} \leftarrow X_{C}^{+}}$is bounded, hence

$$
\left\|\left.\left(\mathcal{L}_{N}^{+}-I\right)\right|_{X_{C}^{+}}\right\|_{X^{+} \leftarrow X_{C}^{+}} \underset{N \rightarrow \infty}{\longrightarrow} 0 .
$$

Assuming hypothesis (H3), this implies

$$
\left\|\left(\mathcal{L}_{N}^{+}-I\right) \mathcal{F}_{s} V^{+}\right\|_{X^{+} \leftarrow X^{+}} \leq\left\|\left(\mathcal{L}_{N}^{+}-I\right) \uparrow_{X_{C}^{+}}\right\|_{X^{+} \leftarrow X_{C}^{+}}\left\|\mathcal{F}_{s} V^{+}\right\|_{X_{C}^{+} \leftarrow X^{+}} \underset{N \rightarrow \infty}{\longrightarrow} 0 .
$$

In particular, there exists a positive integer $N_{0}$ such that, for each integer $N \geq N_{0}$,

$$
\left\|\left(\mathcal{L}_{N}^{+}-I\right) \mathcal{F}_{s} V^{+}\right\|_{X^{+} \leftarrow X^{+}} \leq \frac{1}{2\left\|\left(I-\mathcal{F}_{s} V^{+}\right)^{-1}\right\|_{X^{+} \leftarrow X^{+}}},
$$

i.e., $\left\|\left(\mathcal{L}_{N}^{+}-I\right) \mathcal{F}_{s} V^{+}\right\|_{X^{+} \leftarrow X^{+}}\left\|\left(I-\mathcal{F}_{s} V^{+}\right)^{-1}\right\|_{X^{+} \leftarrow X^{+}} \leq \frac{1}{2}$, which holds since $I-\mathcal{F}_{s} V^{+}$ is invertible with bounded inverse by virtue of hypothesis (H2) and Theorem 2.2. Considering the operator $I-\mathcal{L}_{N}^{+} \mathcal{F}_{s} V^{+}$as a perturbed version of $I-\mathcal{F}_{s} V^{+}$and writing $I-\mathcal{L}_{N}^{+} \mathcal{F}_{s} V^{+}=I-\mathcal{F}_{s} V^{+}-\left(\mathcal{L}_{N}^{+}-I\right) \mathcal{F}_{s} V^{+}$, by the Banach perturbation lemma [46, Theorem 10.1], there exists a positive integer $N_{0}$ such that, for each integer $N \geq N_{0}$, the operator $I-\mathcal{L}_{N}^{+} \mathcal{F}_{s} V^{+}$is invertible and

$$
\begin{aligned}
\left\|\left(I-\mathcal{L}_{N}^{+} \mathcal{F}_{s} V^{+}\right)^{-1}\right\|_{X^{+} \leftarrow X^{+}} & \leq \frac{\left\|\left(I-\mathcal{F}_{s} V^{+}\right)^{-1}\right\|_{X^{+} \leftarrow X^{+}}}{1-\left\|\left(I-\mathcal{F}_{s} V^{+}\right)^{-1}\left(\left(\mathcal{L}_{N}^{+}-I\right) \mathcal{F}_{s} V^{+}\right)\right\|_{X^{+} \leftarrow X^{+}}} \\
& \leq 2\left\|\left(I-\mathcal{F}_{s} V^{+}\right)^{-1}\right\|_{X^{+} \leftarrow X^{+}} .
\end{aligned}
$$

Hence, fixed $\varphi \in X,(4.11)$ has a unique solution $w_{N}^{*} \in X^{+}$. For the same $\varphi$, let $e_{N}^{*} \in$ $X^{+}$such that $w_{N}^{*}=w^{*}+e_{N}^{*}$, where $w^{*} \in X^{+}$is the unique solution of (2.9). Then $w^{*}+e_{N}^{*}=\mathcal{L}_{N}^{+} \mathcal{F}_{s} V\left(\varphi, w^{*}+e_{N}^{*}\right)=\mathcal{L}_{N}^{+} \mathcal{F}_{s} V\left(\varphi, w^{*}\right)+\mathcal{L}_{N}^{+} \mathcal{F}_{s} V^{+} e_{N}^{*}=\mathcal{L}_{N}^{+} w^{*}+\mathcal{L}_{N}^{+} \mathcal{F}_{s} V^{+} e_{N}^{*}$ and $\left(I-\mathcal{L}_{N}^{+} \mathcal{F}_{s} V^{+}\right) e_{N}^{*}=\left(\mathcal{L}_{N}^{+}-I\right) w^{*}$, completing the proof.

4.3. Convergence of the eigenvalues. The proof that the eigenvalues of $T_{M, N}$ approximate those of $T$ follows the lines of the proof for RFDEs in [13], modulo the difference about $V$ mentioned in section 2 and those due to the change of state space. As a consequence, although the proof of the main step (Proposition 4.7) is simplified, the outcome is a stronger result than [13, Proposition 4.5]. Indeed, restricting the state space to a subspace of more regular functions is no longer necessary. This is basically due to the regularizing nature of the right-hand side of (2.2) under hypothesis (H4), which is usually satisfied in applications, as remarked at the end of section 2.

Observe that $T$ and $T_{M, N}$ live on different spaces, which cannot be compared directly because of the different dimensions, viz. infinite vs. finite. In view of this, 
we first translate the problem of studying the eigenvalues of $T_{M, N}$ on $X_{M}$ to that of studying the eigenvalues of finite-rank operators $\hat{T}_{M, N}$ and $\hat{T}_{N}$ on $X$ (Propositions 4.4 and 4.5). Then, in Proposition 4.7, we show that $\hat{T}_{N}$ converges in operator norm to $T$ and, by applying results from spectral approximation theory [22] (Lemma 4.8), we obtain the desired convergence of the eigenvalues of $T_{M, N}$ to the eigenvalues of $T$ (Proposition 4.9 and Theorem 4.10), which represents the main result of the work.

Under some additional hypotheses on the smoothness of the eigenfunctions of $T$, the eigenvalues converge with infinite order. The numerical tests of section 5 show that in practice the infinite order of convergence can be attained. It is reasonable to expect that the regularity of the eigenfunctions depends on the regularity of the model coefficients. A rigorous investigation is ongoing in parallel to the completion of the Floquet theory and more comments are given in section 6 .

Now we introduce the finite-rank operator $\hat{T}_{M, N}$ associated to $T_{M, N}$ and show the relation between their spectra.

Proposition 4.4. The finite-dimensional operator $T_{M, N}$ has the same nonzero eigenvalues, with the same geometric and partial multiplicities, of the operator

$$
\hat{T}_{M, N}:=P_{M} T_{M, N} R_{M \Upsilon_{X_{C}}}: X_{C} \rightarrow X_{C}
$$

Moreover, if $\Phi \in X_{M}$ is an eigenvector of $T_{M, N}$ associated to a nonzero eigenvalue $\mu$, then $P_{M} \Phi \in X_{C}$ is an eigenvector of $\hat{T}_{M, N}$ associated to the same eigenvalue $\mu$.

Proof. Apply [13, Proposition 4.1], since prolongations are polynomials, hence continuous.

Define the operator $\hat{T}_{N}: X \rightarrow X$ as

$$
\hat{T}_{N} \varphi:=V\left(\varphi, w_{N}^{*}\right)_{h},
$$

where $w_{N}^{*} \in X^{+}$is the solution of the fixed point equation (4.11), which, under hypotheses (H1) to (H3), is unique thanks to Propositions 4.2 and 4.3. Observe that $w_{N}^{*}$ is a polynomial, hence, in particular, $w_{N}^{*} \in X_{C}^{+}$. Then, for $\varphi \in X_{C}$, by (4.12),

$$
\begin{aligned}
\hat{T}_{M, N} \varphi & =P_{M} T_{M, N} R_{M} \varphi \\
& =P_{M} R_{M} V\left(P_{M} R_{M} \varphi, P_{N}^{+} W^{*}\right)_{h} \\
& =\mathcal{L}_{M} V\left(\mathcal{L}_{M} \varphi, w_{N}^{*}\right)_{h} \\
& =\mathcal{L}_{M} \hat{T}_{N} \mathcal{L}_{M} \varphi
\end{aligned}
$$

where $W^{*} \in X_{N}^{+}$and $w_{N}^{*} \in X_{C}^{+}$are the solutions, respectively, of (3.5) applied to $\Phi=R_{M} \varphi$ and of (4.11) with $\mathcal{L}_{M} \varphi$ replacing $\varphi$. These solutions are unique under hypotheses (H1) to (H3), thanks again to Propositions 4.2 and 4.3.

Now we show the relation between the spectra of $\hat{T}_{M, N}$ and $\hat{T}_{N}$.

Proposition 4.5. Assume that hypotheses (H1) to (H3) hold and let $M \geq N \geq$ $N_{0}$, with $N_{0}$ given by Proposition 4.3. Then the operator $\hat{T}_{M, N}$ has the same nonzero eigenvalues, with the same geometric and partial multiplicities and associated eigenvectors, of the operator $\hat{T}_{N}$.

Proof. Denote by $\Pi_{r}$ and $\Pi_{r}^{+}$the subspaces of polynomials of degree $r$ of $X$ and $X^{+}$, respectively, and observe that Remark 4.1 applies also here. Note that $w_{N}^{*} \in \Pi_{N-1}^{+}$. 
If $h \geq \tau$, for all $\varphi \in X, \hat{T}_{N} \varphi=V\left(\varphi, w_{N}^{*}\right)_{h} \in \Pi_{N-1}$. Thus both $\hat{T}_{N}$ and $\hat{T}_{M, N}=$ $\mathcal{L}_{M} \hat{T}_{N} \mathcal{L}_{M}$ have range contained in $\Pi_{M}$, being $M \geq N$. By [13, Proposition 4.3 and Remark 4.4], $\hat{T}_{N}$ and $\hat{T}_{M, N}$ have the same nonzero eigenvalues, with the same geometric and partial multiplicities and associated eigenvectors, as their restrictions to $\Pi_{M}$. Observing that $\hat{T}_{M, N} \uparrow_{\Pi_{M}}=\mathcal{L}_{M} \hat{T}_{N} \mathcal{L}_{M} \uparrow_{\Pi_{M}}=\hat{T}_{N} \uparrow_{\Pi_{M}}$, the thesis follows.

Consider now the case $h<\tau$. Denote by $\Pi_{r}^{\mathrm{pw}}$ the subspace of piecewise polynomials of degree $r$ of $X$ on the intervals $\left[\theta^{(q+1)}, \theta^{(q)}\right]$, for $q=0, \ldots, Q-1$. For all $\varphi \in \Pi_{M}^{\mathrm{pw}}, \hat{T}_{N} \varphi=V\left(\varphi, w_{N}^{*}\right)_{h} \in \Pi_{M}^{\mathrm{pw}}$. Let $\mu \neq 0, \varphi \in X$ and $\bar{\varphi} \in \Pi_{M}^{\mathrm{pw}}$ such that $\left(\mu I_{X}-\hat{T}_{N}\right) \varphi=\mu \varphi-V\left(\varphi, w_{N}^{*}\right)_{h}=\bar{\varphi}$. This equation can be rewritten as $\mu \varphi(\theta)=w_{N}^{*}(h+\theta)+\bar{\varphi}(\theta)$ if $\theta \in(-h, 0]$ and as $\mu \varphi(\theta)=\varphi(h+\theta)+\bar{\varphi}(\theta)$ if $\theta \in[-\tau,-h]$. From the first equation, $\varphi$ restricted to $[-h, 0]$ is a polynomial of degree $M$, being $M \geq N$. From the second equation it is easy to show that $\varphi \in \Pi_{M}^{\mathrm{pw}}$ by induction on the intervals $\left[\theta^{(q+1)}, \theta^{(q)}\right]$, for $q=1, \ldots, Q-1$. Hence, by [13, Proposition 4.3], $\hat{T}_{N}$ has the same nonzero eigenvalues, with the same geometric and partial multiplicities and associated eigenvectors, as its restriction to $\Pi_{M}^{\mathrm{pw}}$. The same holds for $\hat{T}_{M, N}=\mathcal{L}_{M} \hat{T}_{N} \mathcal{L}_{M}$ by [13, Proposition 4.3 and Remark 4.4] since its range is contained in $\Pi_{M}^{\mathrm{pw}}$. The thesis

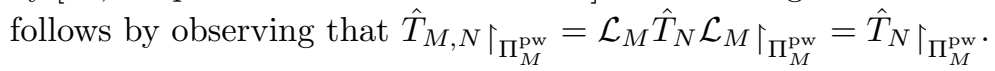

Below we prove the norm convergence of $\hat{T}_{N}$ to $T$, which is the key step to obtain the main result of this work. First we need to extend the results of Theorem 2.2 to $X_{C}^{+}$in the following lemma.

LEMma 4.6. If hypotheses (H2) and (H3) hold, then $\left(I_{X^{+}}-\mathcal{F}_{s} V^{+}\right) \uparrow_{X_{C}^{+}}$is invertible with bounded inverse.

Proof. Since $I_{X^{+}}-\mathcal{F}_{s} V^{+}$is invertible with bounded inverse by virtue of hypothesis (H2) and Theorem 2.2, given $f \in X_{C}^{+}$the equation $\left(I_{X^{+}}-\mathcal{F}_{s} V^{+}\right) w=f$ has a unique solution $w \in X^{+}$, which by hypothesis (H3) is in $X_{C}^{+}$. Hence, the operator $\left(I_{X^{+}}-\mathcal{F}_{s} V^{+}\right) \uparrow_{X_{C}^{+}}$is invertible. It is also bounded, since $\|\cdot\|_{X^{+}} \leq h\|\cdot\|_{X_{C}^{+}}$, which implies $\left\|\mathcal{F}_{s} V^{+} \uparrow_{X_{C}^{+}}\right\|_{X_{C}^{+} \leftarrow X_{C}^{+}} \leq h\left\|\mathcal{F}_{s} V^{+}\right\|_{X_{C}^{+} \leftarrow X^{+}}$. The bounded inverse theorem completes the proof.

Proposition 4.7. If hypotheses (H1) to (H4) hold, then $\left\|\hat{T}_{N}-T\right\|_{X \leftarrow X} \rightarrow 0$ for $N \rightarrow \infty$.

Proof. Let $\varphi \in X$ and let $w^{*}$ and $w_{N}^{*}$ be the solutions of the fixed point equations (2.9) and (4.11), respectively. Recall that $w_{N}^{*}$ is a polynomial. Assuming hypotheses (H3) and (H4) and recalling that $w^{*}=\mathcal{F}_{s} V^{+} w^{*}+\mathcal{F}_{s} V^{-} \varphi$, it is clear that $w^{*} \in X_{C}^{+}$. Hence it follows that $V\left(\varphi, w^{*}\right)_{h} \in X_{C}$ (recall Remark 4.1 and that for $h<\tau$ the space $X_{C}$ is piecewise defined, subsection 4.1). Then $\left(\hat{T}_{N}-T\right) \varphi=$ $V\left(\varphi, w_{N}^{*}\right)_{h}-V\left(\varphi, w^{*}\right)_{h}=V^{+}\left(w_{N}^{*}-w^{*}\right)_{h}$. Assuming also hypotheses (H1) and (H2), by Proposition 4.3, there exists a positive integer $N_{0}$ such that, for any $N \geq N_{0}$,

$$
\begin{aligned}
\left\|\left(\hat{T}_{N}-T\right) \varphi\right\|_{X} & =\left\|V^{+}\left(w_{N}^{*}-w^{*}\right)_{h}\right\|_{X} \\
& \leq\left\|w_{N}^{*}-w^{*}\right\|_{X^{+}} \\
& \leq 2\left\|\left(I_{X^{+}}-\mathcal{F}_{S} V^{+}\right)^{-1}\right\|_{X^{+} \leftarrow X^{+}}\left\|\mathcal{L}_{N}^{+} w^{*}-w^{*}\right\|_{X^{+}} \\
& \leq 2\left\|\left(I_{X^{+}}-\mathcal{F}_{S} V^{+}\right)^{-1}\right\|_{X^{+} \leftarrow X^{+}}\left\|\left(\mathcal{L}_{N}^{+}-I_{X^{+}}\right) \uparrow_{X_{C}^{+}}\right\|_{X^{+} \leftarrow X_{C}^{+}}\left\|w^{*}\right\|_{X_{C}^{+}}
\end{aligned}
$$


holds by virtue of (4.13). Eventually,

$$
\left\|w^{*}\right\|_{X_{C}^{+}} \leq\left\|\left(\left(I_{X^{+}}-\mathcal{F}_{s} V^{+}\right) \uparrow_{X_{C}^{+}}\right)^{-1}\right\|_{X_{C}^{+} \leftarrow X_{C}^{+}}\left\|\mathcal{F}_{s} V^{-}\right\|_{X_{C}^{+} \leftarrow X}\|\varphi\|_{X}
$$

completes the proof thanks to Lemma 4.6 and hypothesis (H4).

The final convergence results rely on a combination of tools from [22], as summarized in the following lemma.

Lemma 4.8. Let $U$ be a Banach space, $A$ a linear and bounded operator on $U$ and $\left\{A_{N}\right\}_{N \in \mathbb{N}}$ a sequence of linear and bounded operators on $U$ such that $\left\|A_{N}-A\right\|_{U \leftarrow U} \rightarrow$ 0 for $N \rightarrow \infty$. If $\mu \in \mathbb{C}$ is an eigenvalue of $A$ with finite algebraic multiplicity $\nu$ and ascent $l$, and $\Delta$ is a neighborhood of $\mu$ such that $\mu$ is the only eigenvalue of $A$ in $\Delta$, then there exists a positive integer $\bar{N}$ such that, for any $N \geq \bar{N}, A_{N}$ has in $\Delta$ exactly $\nu$ eigenvalues $\mu_{N, j}, j \in\{1, \ldots, \nu\}$, counting their multiplicities. Moreover, by setting $\epsilon_{N}:=\left\|\left(A_{N}-A\right)_{\left.\right|_{\mathcal{E}_{\mu}}}\right\|_{U \leftarrow \mathcal{E}_{\mu}}$, where $\mathcal{E}_{\mu}$ is the generalized eigenspace of $\mu$ equipped with the norm $\|\cdot\|_{U}$ restricted to $\mathcal{E}_{\mu}$, the following holds:

$$
\max _{j \in\{1, \ldots, \nu\}}\left|\mu_{N, j}-\mu\right|=O\left(\epsilon_{N}^{1 / l}\right) .
$$

Proof. By [22, Example 3.8 and Theorem 5.22], the norm convergence of $A_{N}$ to $A$ implies the strongly stable convergence $A_{N}-\mu I_{U} \stackrel{s s}{\longrightarrow} A-\mu I_{U}$ for all $\mu$ in the resolvent set of $A$ and all isolated eigenvalues $\mu$ of finite multiplicity of $A$. The thesis follows then by [22, Proposition 5.6 and Theorem 6.7].

Proposition 4.9. Assume that hypotheses (H1) to (H4) hold. If $\mu \in \mathbb{C} \backslash\{0\}$ is an eigenvalue of $T$ with finite algebraic multiplicity $\nu$ and ascent $l$, and $\Delta$ is a neighborhood of $\mu$ such that $\mu$ is the only eigenvalue of $T$ in $\Delta$, then there exists a positive integer $N_{1} \geq N_{0}$, with $N_{0}$ given by Proposition 4.3, such that, for any $N \geq N_{1}, \hat{T}_{N}$ has in $\Delta$ exactly $\nu$ eigenvalues $\mu_{N, j}, j \in\{1, \ldots, \nu\}$, counting their multiplicities. Moreover, if for each $\varphi \in \mathcal{E}_{\mu}$, where $\mathcal{E}_{\mu}$ is the generalized eigenspace of $T$ associated to $\mu$, the function $w^{*}$ that solves (2.9) is of class $C^{p}$, with $p \geq 1$, then

$$
\max _{j \in\{1, \ldots, \nu\}}\left|\mu_{N, j}-\mu\right|=o\left(N^{\frac{1-p}{l}}\right) .
$$

Proof. By Proposition 4.7, $\left\|\hat{T}_{N}-T\right\|_{X \leftarrow X} \rightarrow 0$ for $N \rightarrow \infty$. The first part of the thesis is obtained by applying Lemma 4.8. From the same Lemma 4.8, (4.14) follows with $\epsilon_{N}:=\left\|\left(\hat{T}_{N}-T\right) \uparrow_{\mathcal{E}_{\mu}}\right\|_{X \leftarrow \mathcal{E}_{\mu}}$ and $\mathcal{E}_{\mu}$ the generalized eigenspace of $\mu$ equipped with the norm of $X$ restricted to $\mathcal{E}_{\mu}$.

Let $\varphi_{1}, \ldots, \varphi_{\nu}$ be a basis of $\mathcal{E}_{\mu}$. An element $\varphi$ of $\mathcal{E}_{\mu}$ can be written as $\varphi=$ $\sum_{j=1}^{\nu} \alpha_{j}(\varphi) \varphi_{j}$, with $\alpha_{j}(\varphi) \in \mathbb{C}$, for $j \in\{1, \ldots, \nu\}$, hence

$$
\left\|\left(\hat{T}_{N}-T\right) \varphi\right\|_{X} \leq \max _{j \in\{1, \ldots, \nu\}}\left|\alpha_{j}(\varphi)\right| \sum_{j=1}^{\nu}\left\|\left(\hat{T}_{N}-T\right) \varphi_{j}\right\|_{X} .
$$

The function $\varphi \mapsto \max _{j \in\{1, \ldots, \nu\}}\left|\alpha_{j}(\varphi)\right|$ is a norm on $\mathcal{E}_{\mu}$, so it is equivalent to the norm of $X$ restricted to $\mathcal{E}_{\mu}$. Thus, there exists a positive constant $c$ independent of $\varphi$ such that $\max _{j \in\{1, \ldots, \nu\}}\left|\alpha_{j}(\varphi)\right| \leq c\|\varphi\|_{X}$ and

$$
\epsilon_{N}=\left\|\left(\hat{T}_{N}-T\right) \uparrow_{\mathcal{E}_{\mu}}\right\|\left\|_{X \leftarrow \mathcal{E}_{\mu}} \leq c \sum_{j=1}^{\nu}\right\|\left(\hat{T}_{N}-T\right) \varphi_{j} \|_{X}
$$


Let $j \in\{1, \ldots, \nu\}$. As seen in Proposition 4.7,

$$
\left\|\left(\hat{T}_{N}-T\right) \varphi_{j}\right\|_{X} \leq 2\left\|\left(I_{X^{+}}-\mathcal{F}_{s} V^{+}\right)^{-1}\right\|_{X^{+} \leftarrow X^{+}}\left\|\left(\mathcal{L}_{N}^{+}-I_{X^{+}}\right) w_{j}^{*}\right\|_{X^{+}},
$$

where $w_{j}^{*}$ is the solution of (2.9) associated to $\varphi_{j}$. Now, by well-known results in interpolation theory (see, e.g., [55, Theorems 1.5 and 4.1]), since $w_{j}^{*}$ is of class $C^{p}$, the bound

$$
\begin{aligned}
\left\|\left(\mathcal{L}_{N}^{+}-I_{X^{+}}\right) w_{j}^{*}\right\|_{X^{+}} & \leq h\left(1+\Lambda_{N}\right) E_{N-1}\left(w_{j}^{*}\right) \\
& \leq h\left(1+\Lambda_{N}\right) \frac{6^{p+1} \mathrm{e}^{p}}{1+p}\left(\frac{h}{2}\right)^{p} \frac{1}{(N-1)^{p}} \omega\left(\frac{h}{2(N-1-p)}\right)
\end{aligned}
$$

holds, where $\Lambda_{N}$ is the Lebesgue constant for $\Omega_{N}^{+}, E_{N-1}(\cdot)$ is the best uniform approximation error and $\omega(\cdot)$ is the modulus of continuity of $\left(w_{j}^{*}\right)^{(p)}$ on $[0, h]$. Since hypothesis (H1) is assumed, by classic results on interpolation (see, e.g., [55, Theorem 4.5]), $\Lambda_{N}=o(N)$. Hence, $\epsilon_{N}=o\left(N^{1-p}\right)$ and the thesis follows immediately.

TheOrem 4.10. Assume that hypotheses (H1) to (H4) hold. If $\mu \in \mathbb{C} \backslash\{0\}$ is an eigenvalue of $T$ with finite algebraic multiplicity $\nu$ and ascent $l$, and $\Delta$ is a neighborhood of $\mu$ such that $\mu$ is the only eigenvalue of $T$ in $\Delta$, then there exists a positive integer $N_{1} \geq N_{0}$, with $N_{0}$ given by Proposition 4.3, such that, for any $N \geq N_{1}$ and any $M \geq N, T_{M, N}$ has in $\Delta$ exactly $\nu$ eigenvalues $\mu_{M, N, j}, j \in\{1, \ldots, \nu\}$, counting their multiplicities. Moreover, if for each $\varphi \in \mathcal{E}_{\mu}$, where $\mathcal{E}_{\mu}$ is the generalized eigenspace of $T$ associated to $\mu$, the function $w^{*}$ that solves (2.9) is of class $C^{p}$, with $p \geq 1$, then

$$
\max _{j \in\{1, \ldots, \nu\}}\left|\mu_{M, N, j}-\mu\right|=o\left(N^{\frac{1-p}{l}}\right) .
$$

Proof. If $M \geq N \geq N_{0}$, by Propositions 4.4 and 4.5 the operators $T_{M, N}, \hat{T}_{M, N}$ and $\hat{T}_{N}$ have the same nonzero eigenvalues, with the same geometric and partial multiplicities and associated eigenvectors. The thesis follows by Proposition 4.9.

We conclude this section with a couple of comments. First, nodes other than those required by hypothesis (H1) may be used. Indeed, they are only asked to satisfy the hypotheses of [35, Corollary of Theorem Ia] and $\Lambda_{N}=o(N)$. Let us notice that both are guaranteed by zeros of other families of classic orthogonal polynomials [18]. Anyway, here we assume hypothesis (H1) since these are the nodes we actually use in implementing the method.

Second, in general, it may not be possible to compute exactly the integral in (2.7). If this is the case, an approximation $\tilde{\mathcal{F}}_{s}$ of $\mathcal{F}_{s}$ must be used, leading to a further contribution in the final error. See [14, section 6.3.3] and further comments in Appendix A as far as implementation is concerned.

5. Numerical tests. REs with known solutions and stability properties are rather rare. A notable difficulty is the lack of a characteristic equation for nonautonomous equations, which makes it hard to obtain both theoretical and numerical results to compare with our method. For these reasons, we first compare our method with that of [10] in the autonomous case, where, instead, a characteristic equation can be derived. Then we study a nonlinear equation which possesses a branch of analytically known periodic solutions in a certain range of a varying parameter.

In the following tests we use Chebyshev zeros in $[0, h]$ as $\Omega_{N}^{+}$, as required by hypothesis (H1). In $[-\tau, 0]$ we use Chebyshev extrema as $\Omega_{M}$ if $h \geq \tau$ and as $\Omega_{M}^{(q)}$ for $q \in\{1, \ldots, Q\}$ if $h<\tau$. 

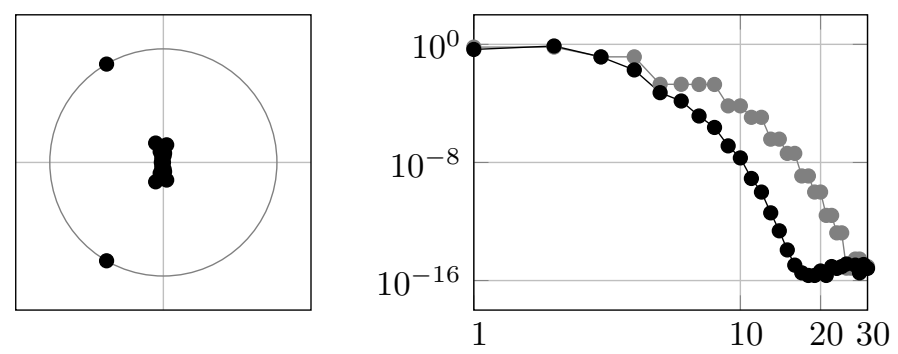

Figure 1. Numerical test with (5.1) where $a=2$ and $\beta=\frac{1}{2} \exp \left(1+\frac{2 \pi}{3 \sqrt{3}}\right)$. Left: eigenvalues of $T(4,0)$ for $M=N=20$ with respect to the unit circle. Right: error with respect to 1 of the absolute value of the dominant eigenvalues of $T(4,0)$ in black and error on the 0 real part of the rightmost characteristic roots obtained with the method of [10] in gray.

Consider the egg cannibalism model

$$
x(t)=\beta \int_{-4}^{-a} x(t+\theta) \mathrm{e}^{-x(t+\theta)} \mathrm{d} \theta,
$$

where $\beta>0$ and $0<a<4$, for which some theoretical results are known [10, section 5.1]. By linearizing it around the nontrivial equilibrium $\log (\beta(4-a))$, we obtain the linear equation

$$
x(t)=\frac{1-\log (\beta(4-a))}{4-a} \int_{-4}^{-a} x(t+\theta) \mathrm{d} \theta .
$$

It corresponds to $(2.2)$ by setting $C(t, \theta):=\frac{1-\log (\beta(4-a))}{4-a}$ for $\theta \in[-\tau,-a], C(t, \theta):=0$ for $\theta \in(-a, 0]$ and $\tau:=4$. Observe that $C(t, \theta)$ is independent of $t$ and piecewise constant in $\theta$, thus making (5.1) an instance of (2.11) with $p=2, \tau_{1}=a$ and $\tau_{2}=4$. By studying the characteristic equation it is known that the equilibrium undergoes a Hopf bifurcation for $a=2$ and $\beta=\frac{1}{2} \exp \left(1+\frac{2 \pi}{3 \sqrt{3}}\right)$, hence the operator $T(h, 0)$ has a complex conjugate pair on the unit circle as its dominant eigenvalues, independently of $h>0$. In this test we choose $h=\tau(=4)$. Figure 1 shows the eigenvalues of $T(4,0)$ for $M=N=20$ and the errors with respect to 1 of the absolute value of the dominant eigenvalues as $M=N$ varies from 1 to 30 , compared with the errors on the 0 real part of the characteristic roots obtained with the method of [10]. Observe that the latter approximates the eigenvalues $\lambda$ of the infinitesimal generator (characteristic roots), which are related to the eigenvalues $\mu$ of $T$ (characteristic multipliers) by $\mu=\mathrm{e}^{\lambda h}$. Notice that both methods experiment the proved convergence of infinite order, with apparently larger error constants for the method of [10].

The second numerical test is based on the nonlinear equation

$$
x(t)=\frac{\gamma}{2} \int_{-3}^{-1} x(t+\theta)(1-x(t+\theta)) \mathrm{d} \theta,
$$

linearized around the periodic solution

$$
\bar{x}(t)=\frac{1}{2}+\frac{\pi}{4 \gamma}+\sqrt{\frac{1}{2}-\frac{1}{\gamma}-\frac{\pi}{2 \gamma^{2}}\left(1+\frac{\pi}{4}\right)} \sin \left(\frac{\pi}{2} t\right),
$$



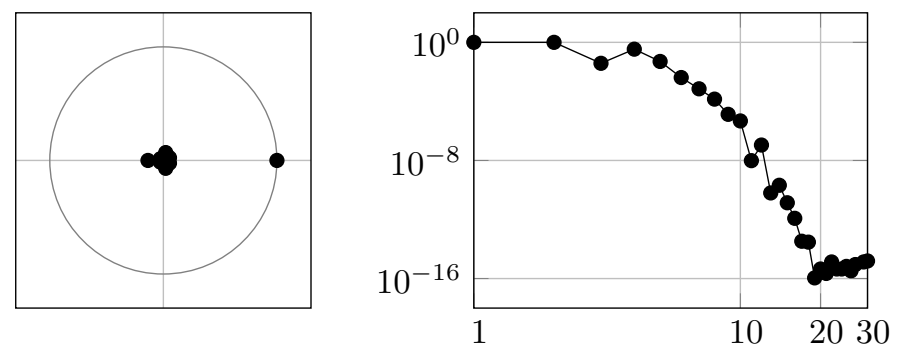

Figure 2. Numerical test with (5.2) where $\gamma=4$, linearized around (5.3). Left: eigenvalues of $T(4,0)$ for $M=N=20$ with respect to the unit circle. Right: error on the known eigenvalue 1 of $T(4,0)$.
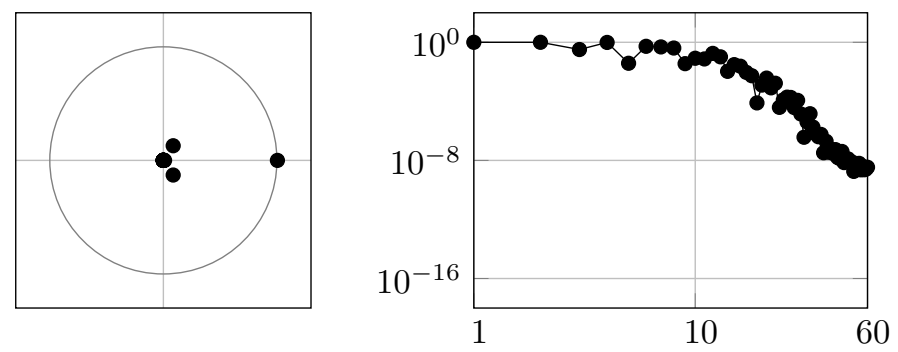

Figure 3. Numerical test with (5.2) where $\gamma=4.4$, linearized around a numerically approximated periodic solution of period $\Omega \approx 8.0189$. Left: eigenvalues of $T(\Omega, 0)$ for $M=N=20$ with respect to the unit circle. Right: error on the known eigenvalue 1 of $T(\Omega, 0)$.

which exists for $\gamma \geq 2+\frac{\pi}{2}$ and has period 4 [9]. The linearized equation reads

$$
x(t)=\frac{\gamma}{2} \int_{-3}^{-1}(1-2 \bar{x}(t+\theta)) x(t+\theta) \mathrm{d} \theta,
$$

which corresponds to $(2.2)$ by setting $C(t, \theta):=\frac{\gamma}{2}(1-2 \bar{x}(t+\theta))$ for $\theta \in[-\tau,-1]$, $C(t, \theta):=0$ for $\theta \in(-1,0]$ and $\tau:=3$. Observe that $C(t, \theta)$ is continuous in $t$ and for each $t$ it may have a single discontinuity in $\theta$, thus adhering to (2.11) with $p=2, \tau_{1}=1$ and $\tau_{2}=3$. Although not much is known theoretically about stability, the monodromy operator $T(4,0)$ has always an eigenvalue 1 due to the linearization around the periodic solution, which allows us to test the accuracy of the approximation. Figure 2 shows the eigenvalues of $T(4,0)$ and the errors on the known eigenvalue 1 for $\gamma=4$. By using standard zero-finding routines (e.g., MATLAB's fzero), we can detect for $\gamma \approx 4.3247$ an eigenvalue crossing the unit circle outwards through -1 , which characterizes a period doubling bifurcation. The branch of periodic solutions arising from the latter is not known analytically. In [9] these periodic solutions are computed numerically by adapting the method of [32] for RFDEs or of [49] for differential algebraic equations with delays (see relevant comments in section 6). The method is then applied to the equation linearized around the numerical solution. Figure 3 shows the eigenvalues of $T(\Omega, 0)$ and the errors on the known eigenvalue 1 for $\gamma=4.4$, where $\Omega \approx 8.0189$ is the computed period of the numerically approximated periodic solution. Notice again that our method works equally well, independently of the relation between $\Omega$ and $\tau$.

It can be seen that to achieve the same accuracy as for the branch of periodic 
solutions (5.3), a number of nodes more than double than before must be used. This fact is in line with usual properties of pseudospectral methods, which exhibit slower convergence as the length of the discretization interval increases (although the infinite order is preserved). Indeed, by standard results on interpolation, the error depends both on the length of the interpolation interval and on bounds on the derivatives of the interpolated function: in this case, after the period doubling bifurcation both the period of the solution (length of the interpolation interval) and the number of oscillations (related to the magnitude of the derivatives) are roughly double than before. Observe, however, that here the error takes also into account for the error in the computation of the reference solution.

6. Future perspectives. In this work we propose a numerical method to approximate the spectrum of evolution operators for linear REs. This concluding section contains diverse comments on open problems and possible future research lines, most of which were briefly touched along the text.

The numerical experiments suggest that the order of convergence of the approximated eigenvalues to the exact ones is infinite and Theorem 4.10 guarantees that this is the case if the eigenfunctions of the evolution operator are sufficiently smooth. Although it is reasonable to expect that any desired regularity of the eigenfunctions can be achieved by imposing suitable conditions on $C(t, \theta)$ (see, e.g., [54] for some results in this direction for convolution products), this has not been proved yet and remains an open question that the authors are investigating.

Regarding the application to the asymptotic stability of periodic solutions of nonlinear autonomous REs, another open problem is the validity of a Floquet theory for linear periodic REs and of a corresponding principle of linearized stability. In view of [25], this would be guaranteed by the validity of assumptions $(\mathrm{F}),(\mathrm{H})$ and $(\Xi)$ of [31, section XIV.4]. A preliminary study reveals that assumption (F) should be guaranteed by suitable regularity assumptions on $C(t, \theta)$. On the other hand, some results on the regularity of Volterra integrals, similar to the ones mentioned above with respect to the regularity of eigenfunctions, seem to be needed for assumptions $(\mathrm{H})$ and $(\Xi)$. Investigating these details and thus proving the validity of a Floquet theory is an ongoing effort by the authors and colleagues.

As mentioned in section 2, the discretization proposed in this work can be used in principle in the framework of [15] to compute Lyapunov exponents for generic solutions of nonautonomous REs. Numerical tests on this approach appear in [9] with promising results. Investigating this natural development is in the future plans of the authors. Indeed, it goes beyond the scopes of the present paper since it requires to work in a Hilbert rather than in a Banach setting. Incidentally, notice how this change would require a restriction of the state space, as opposed to RFDEs in [15].

In the literature of population dynamics, the recent paper [26] deals with a model based on retarded functional equations containing also point evaluation terms, i.e., Volterra integrals with kernel of Dirac type. The presence of these terms may give rise to neutral dynamics, adding several difficulties both to the theoretical treatment (they are not covered in general by $[25,31]$ ) and to the proof of convergence of the numerical method (the regularization effect on the solutions, essential to the current proof, is not guaranteed and in general does not take place). Anyway, investigating the neutral case remains in the interests of the authors.

Finally, in structured population models, REs are often coupled with RFDEs (see, e.g., [29, 50]). Extending the method to such coupled equations, as in the case of $[10,11]$ for equilibria, poses additional and nontrivial difficulties in proving the 
convergence of the approximated eigenvalues, with respect to both the RFDE case of [13] and the RE case of the present work. In fact, due to the coupling, there is a delicate interplay between the diverse regularization mechanisms, with different consequences on the two components of the solution. With respect to the regularity of eigenfunctions and to the validity of a Floquet theory, coupled equations retain the same difficulties as outlined above for REs and may be addressed by similar solutions, as it appears reasonable. The extension of the method to coupled equations, including a rigorous convergence proof and error analysis, together with numerical tests, is the subject of a distinct paper in preparation by the authors. Nevertheless, in the nonlinear context and for practical applications, this approach inevitably relies on the computation of the relevant periodic solutions. In this sense, an extension of [32] is being developed by the authors and colleagues. The final objective of these research lines is the study of the dynamics of the realistic Daphnia model of [29], which brings in several nontrivial challenges beyond those related to the discretization of the evolution operators.

Appendix A. Matrix representation. In this appendix we describe the explicit construction of a matrix representing the discretization of the evolution operator (2.4) according to (3.6). The reference is to model (2.11). We start by introducing some notations for block matrices.

If $h \geq \tau$, for $\Phi \in X_{M}$ and $m \in\{0, \ldots, M\}$, denote $\left(\Phi_{d m+1}, \ldots, \Phi_{d(m+1)}\right)$, i.e., the $(m+1)$-th $d$-sized block of components of $\Phi$, as $[\Phi]_{m}$. If $h<\tau$, instead, for $\Phi \in X_{M}, q \in\{1, \ldots, Q\}$ and $m \in\{0, \ldots, M-1\}$ and for $q=Q$ and $m=M$, denote $\left(\Phi_{d((q-1) M+m)+1}, \ldots, \Phi_{d((q-1) M+m+1)}\right)$, i.e., the $(m+1)$-th $d$-sized block of components of the $q$-th block of $\Phi$, as $[\Phi]_{q, m}$. Finally, for $W \in X_{N}^{+}$and $n \in\{1, \ldots, N\}$, denote $\left(W_{d(n-1)+1}, \ldots, W_{d n}\right)$, i.e., the $n$-th $d$-sized block of components of $W$, as $[W]_{n}$.

In the following, 0 denotes the scalar zero or a matrix of zeros of the dimensions implied by the context.

A.1. The matrix $T_{M}^{(1)}$. Let $\Phi \in X_{M}$. If $h>\tau$, for $m \in\{0, \ldots, M\}\left[T_{M}^{(1)} \Phi\right]_{m}=$ $\left(V^{-} P_{M} \Phi\right)_{h}\left(\theta_{M, m}\right)=V^{-} P_{M} \Phi\left(h+\theta_{M, m}\right)=0$, hence $T_{M}^{(1)}=0 \in \mathbb{R}^{d(M+1) \times d(M+1)}$. If $h=\tau$, instead, for $m \in\{0, \ldots, M-1\},\left[T_{M}^{(1)} \Phi\right]_{m}=0$ as above. For $m=M$, $\left[T_{M}^{(1)} \Phi\right]_{M}=V^{-} P_{M} \Phi\left(h+\theta_{M, M}\right)=P_{M} \Phi\left(\theta_{M, 0}\right)=\Phi_{0}$. Thus

$$
T_{M}^{(1)}=\left(\begin{array}{cccc}
0 & 0 & \cdots & 0 \\
\vdots & \vdots & \ddots & \vdots \\
0 & 0 & \cdots & 0 \\
1 & 0 & \cdots & 0
\end{array}\right) \otimes I_{d} \in \mathbb{R}^{d(M+1) \times d(M+1)}
$$

Finally, if $h<\tau$, for $m \in\{0, \ldots, M-1\}$ and $q \in\{1, \ldots, Q-1\}$,

$$
\left[T_{M}^{(1)} \Phi\right]_{q, m}=V^{-} P_{M} \Phi\left(h+\theta_{M, m}^{(q)}\right)= \begin{cases}0, & q=1, \\ P_{M} \Phi\left(\theta_{M, m}^{(q-1)}\right)=\Phi_{m}^{(q-1)}, & q \in\{2, \ldots, Q-1\}\end{cases}
$$

while for $m \in\{0, \ldots, M\}$ and $q=Q$,

$$
\left[T_{M}^{(1)} \Phi\right]_{Q, m}=P_{M} \Phi\left(h+\theta_{M, m}^{(Q)}\right)=\sum_{j=0}^{M} \ell_{M, j}^{(Q-1)}\left(h+\theta_{M, m}^{(Q)}\right) \Phi_{j}^{(Q-1)} .
$$


Observe that if $Q h=\tau$, then $\left[T_{M}^{(1)} \Phi\right]_{Q, m}=\Phi_{m}^{(Q-1)}$, since $h+\theta_{M, m}^{(Q)}=\theta_{M, m}^{(Q-1)}$. Then $T_{M}^{(1)} \in \mathbb{R}^{d(Q M+1) \times d(Q M+1)}$ is given by

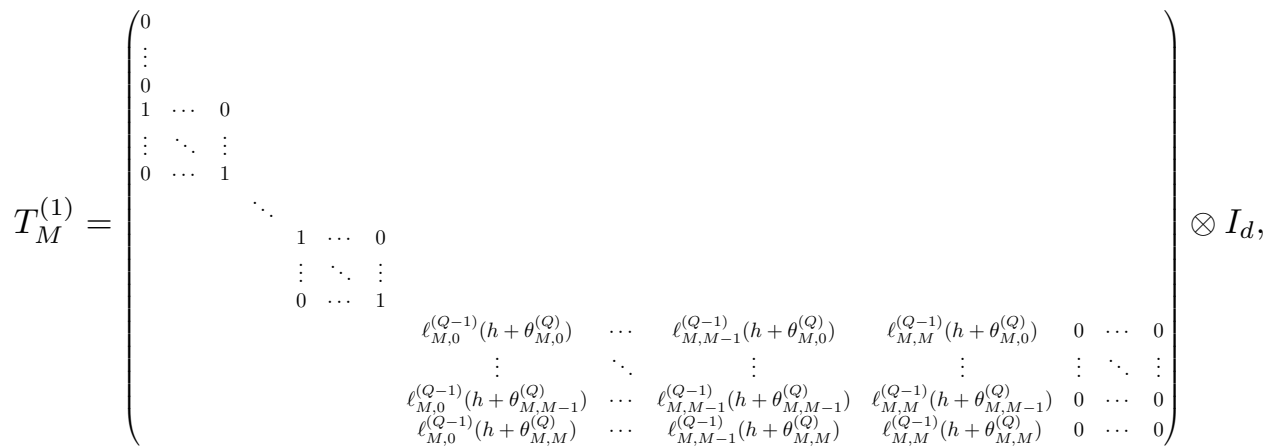

where missing entries are 0 . The order of rows and columns corresponds to the order of components in (3.3). Indeed it can be seen as a block matrix with $Q$ rows (respectively, columns), where the first $Q-1$ consist of blocks of height (respectively, width) $M$ and the last of blocks of height (respectively, width) $M+1$. However, looking at the actual matrix, a slightly different block structure emerges: still $Q-1$ rows of height $M$ and a last row of height $M+1$ can be seen, but there appear $Q-2$ columns of width $M$ followed by a column of width $M+1$ and a last column of width $M$; the top-left column (of zeros) has height $M$, the identity blocks are $I_{M}$, the block of Lagrange coefficients has dimensions $(M+1) \times(M+1)$ and the bottom-right block of zeros has dimensions $(M+1) \times M$. Note that if $Q h=\tau$ then $\ell_{M, j}^{(Q-1)}\left(h+\theta_{M, m}^{(Q)}\right)=\ell_{M, j}^{(Q-1)}\left(\theta_{M, m}^{(Q-1)}\right)=\delta_{m, j}$ and the block of Lagrange coefficients is actually $I_{M+1}$.

Let us notice that in the MATLAB codes the Lagrange coefficients (appearing here and in the sequel) are evaluated by resorting to barycentric interpolation [6].

A.2. The matrix $T_{M, N}^{(2)}$. Let $W \in X_{N}^{+}$. If $h>\tau$, for $m \in\{0, \ldots, M\}$,

$$
\left[T_{M, N}^{(2)} W\right]_{m}=\left(V^{+} P_{N}^{+} W\right)_{h}\left(\theta_{M, m}\right)=P_{N}^{+} W\left(h+\theta_{M, m}\right)=\sum_{n=1}^{N} \ell_{N, n}^{+}\left(h+\theta_{M, m}\right) W_{n},
$$

hence

$$
T_{M, N}^{(2)}=\left(\begin{array}{ccc}
\ell_{N, 1}^{+}\left(h+\theta_{M, 0}\right) & \cdots & \ell_{N, N}^{+}\left(h+\theta_{M, 0}\right) \\
\vdots & \ddots & \vdots \\
\ell_{N, 1}^{+}\left(h+\theta_{M, M}\right) & \cdots & \ell_{N, N}^{+}\left(h+\theta_{M, M}\right)
\end{array}\right) \otimes I_{d} \in \mathbb{R}^{d(M+1) \times d N} .
$$

767 If $h=\tau$, instead, for $m \in\{0, \ldots, M-1\}$, as above,

$$
\left[T_{M, N}^{(2)} W\right]_{m}=\sum_{n=1}^{N} \ell_{N, n}^{+}\left(h+\theta_{M, m}\right) W_{n},
$$


while for $m=M,\left[T_{M, N}^{(2)} W\right]_{M}=V^{+} P_{N}^{+} W\left(h+\theta_{M, M}\right)=V^{+} P_{N}^{+} W(0)=0$. Thus

$$
T_{M, N}^{(2)}=\left(\begin{array}{ccc}
\ell_{N, 1}^{+}\left(h+\theta_{M, 0}\right) & \cdots & \ell_{N, N}^{+}\left(h+\theta_{M, 0}\right) \\
\vdots & \ddots & \vdots \\
\ell_{N, 1}^{+}\left(h+\theta_{M, M-1}\right) & \cdots & \ell_{N, N}^{+}\left(h+\theta_{M, M-1}\right) \\
0 & \cdots & 0
\end{array}\right) \otimes I_{d} \in \mathbb{R}^{d(M+1) \times d N} .
$$

Finally, if $h<\tau$, for $m \in\{0, \ldots, M-1\}$ and $q \in\{1, \ldots, Q\}$,

$$
\left[T_{M, N}^{(2)} W\right]_{q, m}=V^{+} P_{N}^{+} W\left(h+\theta_{M, m}^{(q)}\right)= \begin{cases}\sum_{n=1}^{N} \ell_{N, n}^{+}\left(h+\theta_{M, m}^{(q)}\right) W_{n}, & q=1, \\ 0, & q \in\{2, \ldots, Q\},\end{cases}
$$

and $\left[T_{M, N}^{(2)} W\right]_{Q, M}=V^{+} P_{N}^{+} W\left(h+\theta_{M, M}^{(Q)}\right)=V^{+} P_{N}^{+} W(h-\tau)=0$. Then

$$
T_{M, N}^{(2)}=\left(\begin{array}{ccc}
\ell_{N, 1}^{+}\left(h+\theta_{M, 0}^{(1)}\right) & \cdots & \ell_{N, N}^{+}\left(h+\theta_{M, 0}^{(1)}\right) \\
\vdots & \ddots & \vdots \\
\ell_{N, 1}^{+}\left(h+\theta_{M, M-1}^{(1)}\right) & \cdots & \ell_{N, N}^{+}\left(h+\theta_{M, M-1}^{(1)}\right) \\
0 & \cdots & 0 \\
\vdots & \ddots & \vdots \\
0 & \cdots & 0
\end{array}\right) \otimes I_{d} \in \mathbb{R}^{d(Q M+1) \times d N}
$$

A.3. The matrix $U_{M, N}^{(1)}$. Let $\Phi \in X_{M}$ and, for $t>0$, define

$$
\kappa(t):=\max _{k \in\{0, \ldots, p\}}\left\{\tau_{k}<t\right\} .
$$

Note that $\kappa$ is nondecreasing. For $n \in\{1, \ldots, N\}$,

$$
\left[U_{M, N}^{(1)} \Phi\right]_{n}=\mathcal{F}_{s} V^{-} P_{M} \Phi\left(t_{N, n}\right)=\sum_{k=1}^{p} \int_{-\tau_{k}}^{-\tau_{k-1}} C_{k}\left(s+t_{N, n}, \theta\right) V^{-} P_{M} \Phi\left(t_{N, n}+\theta\right) \mathrm{d} \theta .
$$

If $h \geq \tau$, define also

$$
\hat{N}:= \begin{cases}0, & t_{N, n}>\tau \text { for all } n \in\{1, \ldots, N\}, \\ \max _{n \in\{1, \ldots, N\}}\left\{t_{N, n} \leq \tau\right\}, & \text { otherwise. }\end{cases}
$$

Hence, for $n \in\{1, \ldots, \hat{N}\}$ (if $\hat{N} \neq 0$ ),

$$
\begin{aligned}
{\left[U_{M, N}^{(1)} \Phi\right]_{n}=} & \int_{-\tau_{\kappa\left(t_{N, n}\right)+1}}^{-t_{N, n}} C_{\kappa\left(t_{N, n}\right)+1}\left(s+t_{N, n}, \theta\right) \sum_{m=0}^{M} \ell_{M, m}\left(t_{N, n}+\theta\right) \Phi_{m} \mathrm{~d} \theta \\
& +\sum_{k=\kappa\left(t_{N, n}\right)+2}^{p} \int_{-\tau_{k}}^{-\tau_{k-1}} C_{k}\left(s+t_{N, n}, \theta\right) \sum_{m=0}^{M} \ell_{M, m}\left(t_{N, n}+\theta\right) \Phi_{m} \mathrm{~d} \theta
\end{aligned}
$$


784

and, for $n \in\{\hat{N}+1, \ldots, N\},\left[U_{M, N}^{(1)} \Phi\right]_{n}=0$. Observe that the first integral in (A.2) may be zero. For $m \in\{0, \ldots, M\}$ and $n \in\{1, \ldots, \hat{N}\}$ (if $\hat{N} \neq 0$ ), let

$$
\begin{aligned}
\mathbb{R}^{d \times d} \ni \Theta_{n, m}:= & \int_{-\tau_{\kappa\left(t_{N, n}\right)+1}}^{-t_{N, n}} C_{\kappa\left(t_{N, n}\right)+1}\left(s+t_{N, n}, \theta\right) \ell_{M, m}\left(t_{N, n}+\theta\right) \mathrm{d} \theta \\
& +\sum_{k=\kappa\left(t_{N, n}\right)+2}^{p} \int_{-\tau_{k}}^{-\tau_{k-1}} C_{k}\left(s+t_{N, n}, \theta\right) \ell_{M, m}\left(t_{N, n}+\theta\right) \mathrm{d} \theta .
\end{aligned}
$$

Then

$$
U_{M, N}^{(1)}=\left(\begin{array}{ccc}
\Theta_{1,0} & \cdots & \Theta_{1, M} \\
\vdots & \ddots & \vdots \\
\Theta_{\hat{N}, 0} & \cdots & \Theta_{\hat{N}, M} \\
0 & \cdots & 0 \\
\vdots & \ddots & \vdots \\
0 & \cdots & 0
\end{array}\right) \in \mathbb{R}^{d N \times d(M+1)}
$$

which is the zero matrix if $\hat{N}=0$.

If $h<\tau$, instead, for $n \in\{1, \ldots, N\}$ and $q \in\{0, \ldots, Q-1\}$, define $t_{N, n}^{(q)}=q h+$ $t_{N, n}$. Observe that, for $q \in\{1, \ldots, Q-1\},\left[t_{N, n}-\tau_{k}, t_{N, n}-\tau_{k-1}\right] \cap(-q h,-(q-1) h] \neq \emptyset$ if and only if $\kappa\left(t_{N, n}^{(q-1)}\right)+1 \leq k \leq \kappa\left(t_{N, n}^{(q)}\right)+1$ and $\left[t_{N, n}-\tau_{k}, t_{N, n}-\tau_{k-1}\right] \cap[-\tau,-(Q-$ $1) h] \neq \emptyset$ if and only if $k \geq \kappa\left(t_{N, n}^{(Q-1)}\right)+1$. Observe also that $\kappa\left(t_{N, n}^{(q-1)}\right)$ and $\kappa\left(t_{N, n}^{(q)}\right)$ may be equal. For $n \in\{1, \ldots, N\}, k \in\{1, \ldots, p\}$ and $q \in\{1, \ldots, Q-1\}$, define

$$
\begin{aligned}
a_{k, q} & :=\max \left\{-\tau_{k},-t_{N, n}^{(q)}\right\}, & a_{k, Q} & :=-\tau_{k}, \\
b_{k, q} & :=\min \left\{-\tau_{k-1},-t_{N, n}^{(q-1)}\right\}, & b_{k, Q} & :=\min \left\{-\tau_{k-1},-t_{N, n}^{(Q-1)}\right\}, \\
\kappa_{n, q} & :=\min \left\{\kappa\left(t_{N, n}^{(q)}\right)+1, p\right\}, & \kappa_{n, Q} & :=p .
\end{aligned}
$$

Then, for $n \in\{1, \ldots, N\}$,

$$
\left[U_{M, N}^{(1)} \Phi\right]_{n}=\sum_{q=1}^{Q} \sum_{k=\kappa\left(t_{N, n}^{(q-1)}\right)+1}^{\kappa_{n, q}} \int_{a_{k, q}}^{b_{k, q}} C_{k}\left(s+t_{N, n}, \theta\right) \sum_{m=0}^{M} \ell_{M, m}^{(q)}\left(t_{N, n}+\theta\right) \Phi_{m}^{(q)} \mathrm{d} \theta,
$$

with the convention that $\sum_{k=k_{1}}^{k_{2}} a_{k}=0$ if $k_{2}<k_{1}$. Observe that some of the integrals may be zero. For $n \in\{1, \ldots, N\}, m \in\{0, \ldots, M\}$ and $q \in\{1, \ldots, Q\}$, define

$$
\mathbb{R}^{d \times d} \ni \Theta_{n, m}^{(q)}:=\sum_{k=\kappa\left(t_{N, n}^{(q-1)}\right)+1}^{\kappa_{n, q}} \int_{a_{k, q}}^{b_{k, q}} C_{k}\left(s+t_{N, n}, \theta\right) \ell_{M, m}^{(q)}\left(t_{N, n}+\theta\right) \mathrm{d} \theta
$$

and recall that, for $q \in\{1, \ldots, Q-1\}, \Phi_{M}^{(q)}=\Phi_{0}^{(q+1)}$. Then $U_{M, N}^{(1)} \in \mathbb{R}^{d N \times d(Q M+1)}$ is given by

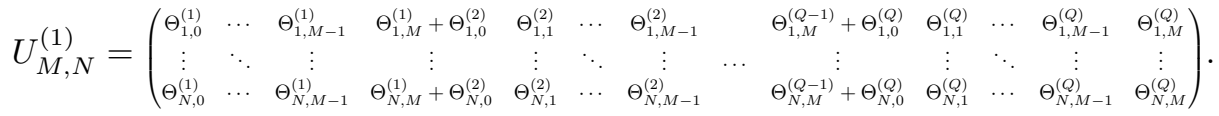


Eventually, with reference to the last comment of section 4, the various integrals appearing in the construction of the elements of $U_{M, N}^{(1)}$ should be computed with a quadrature formula that, in presence of sufficient regularity of the model coefficients, preserves the infinite order of convergence of Theorem 4.10. The same remark holds for the elements of $U_{N}^{(2)}$ in Appendix A.4. Specifically, in the MATLAB codes we resort to Clenshaw-Curtis quadrature [60].

A.4. The matrix $U_{N}^{(2)}$. Let $W \in X_{N}^{+}$. Define $\kappa(t)$ as in (A.1), for $t>0$. For $n \in\{1, \ldots, N\}$,

$$
\begin{aligned}
{\left[U_{N}^{(2)} W\right]_{n}=} & \mathcal{F}_{s} V^{+} P_{N}^{+} W\left(t_{N, n}\right) \\
= & \sum_{k=1}^{p} \int_{-\tau_{k}}^{-\tau_{k-1}} C_{k}\left(s+t_{N, n}, \theta\right) V^{+} P_{N}^{+} W\left(t_{N, n}+\theta\right) \mathrm{d} \theta \\
= & \sum_{k=1}^{\kappa\left(t_{N, n}\right)} \int_{-\tau_{k}}^{-\tau_{k-1}} C_{k}\left(s+t_{N, n}, \theta\right) \sum_{i=1}^{N} \ell_{N, i}^{+}\left(t_{N, n}+\theta\right) W_{i} \mathrm{~d} \theta \\
& \quad+\int_{-\tau_{\kappa\left(t_{N, n}\right)}} C_{\min \left\{\kappa\left(t_{N, n}\right)+1, p\right\}}\left(s+t_{N, n}, \theta\right) \sum_{i=1}^{N} \ell_{N, i}^{+}\left(t_{N, n}+\theta\right) W_{i} \mathrm{~d} \theta
\end{aligned}
$$

with the convention that $\sum_{k=k_{1}}^{k_{2}} a_{k}=0$ if $k_{2}<k_{1}$. Observe that the last integral may be zero. For $n \in\{1, \ldots, N\}$ and $i \in\{1, \ldots, N\}$, let

$$
\mathbb{R}^{d \times d} \ni \Gamma_{n, i}:=\sum_{k=1}^{\kappa\left(t_{N, n}\right)} \int_{-\tau_{k}}^{-\tau_{k-1}} C_{k}\left(s+t_{N, n}, \theta\right) \ell_{N, i}^{+}\left(t_{N, n}+\theta\right) \mathrm{d} \theta
$$

$$
+\int_{-\min \left\{t_{N, n}, \tau\right\}}^{-\tau_{\kappa\left(t_{N, n}\right)}} C_{\min \left\{\kappa\left(t_{N, n}\right)+1, p\right\}}\left(s+t_{N, n}, \theta\right) \ell_{N, i}^{+}\left(t_{N, n}+\theta\right) \mathrm{d} \theta .
$$

Then

$$
U_{N}^{(2)}=\left(\begin{array}{ccc}
\Gamma_{1,1} & \cdots & \Gamma_{1, N} \\
\vdots & \ddots & \vdots \\
\Gamma_{N, 1} & \cdots & \Gamma_{N, N}
\end{array}\right) \in \mathbb{R}^{d N \times d N}
$$

\section{REFERENCES}

[1] DDE-BIFTOOL, http://ddebiftool.sourceforge.net/.

[2] A. Bellen and M. Zennaro, Numerical Methods for Delay Differential Equations, Numer. Math. Sci. Comput., Clarendon Press, 2003.

[3] R. E. Bellman, On the computational solution of differential-difference equations, J. Math. Anal. Appl., 2 (1961), pp. 108-110, https://doi.org/10.1016/0022-247X(61)90049-X.

[4] R. E. Bellman and K. L. Cooke, Differential-Difference Equations, Academic Press, 1963.

[5] R. E. Bellman And K. L. Cooke, On the computational solution of differential-difference equations, J. Math. Anal. Appl., 12 (1965), pp. 495-500, https://doi.org/10.1016/ 0022-247X(65)90017-X.

[6] J.-P. Berrut and L. N. Trefethen, Barycentric Lagrange interpolation, SIAM Rev., 46 (2004), pp. 501-517, https://doi.org/10.1137/S0036144502417715. 
[7] D. Breda, O. Diekmann, W. F. de Graaf, A. Pugliese, and R. Vermiglio, On the formulation of epidemic models (an appraisal of Kermack and McKendrick), J. Biol. Dyn., 6 (2012), pp. 103-117, https://doi.org/10.1080/17513758.2012.716454.

[8] D. Breda, O. Diekmann, M. Gyllenberg, F. Scarabel, and R. Vermiglio, Pseudospectral discretization of nonlinear delay equations: New prospects for numerical bifurcation analysis, SIAM J. Appl. Dyn. Syst., 15 (2016), pp. 1-23, https://doi.org/10.1137/15M1040931.

[9] D. Breda, O. Diekmann, D. Liessi, and F. Scarabel, Numerical bifurcation analysis of a class of nonlinear renewal equations, Electron. J. Qual. Theory Differ. Equ., 65 (2016), pp. 1-24, https://doi.org/10.14232/ejqtde.2016.1.65.

[10] D. Breda, O. Diekmann, S. Maset, and R. Vermiglio, A numerical approach for investigating the stability of equilibria for structured population models, J. Biol. Dyn., 7 (2013), pp. 4-20, https://doi.org/10.1080/17513758.2013.789562.

[11] D. Breda, P. Getto, J. SÁnchez Sanz, and R. Vermiglio, Computing the eigenvalues of realistic Daphnia models by pseudospectral methods, SIAM J. Sci. Comput., 37 (2015), pp. A2607-A2629, https://doi.org/10.1137/15M1016710.

[12] D. Breda, S. Maset, And R. Vermiglio, Pseudospectral differencing methods for characteristic roots of delay differential equations, SIAM J. Sci. Comput., 27 (2005), pp. 482-495, https://doi.org/10.1137/030601600.

[13] D. Breda, S. Maset, and R. Vermiglio, Approximation of eigenvalues of evolution operators for linear retarded functional differential equations, SIAM J. Numer. Anal., 50 (2012), pp. 1456-1483, https://doi.org/10.1137/100815505.

[14] D. Breda, S. Maset, and R. Vermiglio, Stability of Linear Delay Differential Equations: A Numerical Approach with MATLAB, SpringerBriefs in Control, Automation and Robotics, Springer-Verlag, New York, 2015, https://doi.org/10.1007/978-1-4939-2107-2.

[15] D. Breda And E. VAN Vleck, Approximating Lyapunov exponents and Sacker-Sell spectrum for retarded functional differential equations, Numer. Math., 126 (2014), pp. 225-257, https://doi.org/10.1007/s00211-013-0565-1.

[16] H. Brunner, Collocation Methods for Volterra Integral and Related Functional Differential Equations, no. 15 in Cambridge Monogr. Appl. Comput. Math., Cambridge University Press, Cambridge, 2004.

[17] H. Brunner, Volterra Integral Equations: An Introduction to Theory and Applications, no. 30 in Cambridge Monogr. Appl. Comput. Math., Cambridge University Press, Cambridge, 2017.

[18] L. Brutman, Lebesgue functions for polynomial interpolation - a survey, Annals of Numerical Mathematics, 4 (1997), pp. 111-127.

[19] E. BUElER, Error bounds for approximate eigenvalues of periodic-coefficient linear delay differential equations, SIAM J. Numer. Anal., 45 (2007), pp. 2510-2536, https://doi.org/10. $1137 / 050633330$.

[20] E. A. Butcher And O. A. Bobrenkov, On the Chebyshev spectral continuous time approximation for constant and periodic delay differential equations, Commun. Nonlinear Sci. Numer. Simul., 16 (2011), pp. 1541-1554, https://doi.org/10.1016/j.cnsns.2010.05.037.

[21] E. A. Butcher, H. Ma, E. Bueler, V. Averina, and Z. Szabó, Stability of linear timeperiodic delay-differential equations via Chebyshev polynomials, Internat. J. Numer. Methods Engrg., 59 (2004), pp. 895-922, https://doi.org/10.1002/nme.894.

[22] F. Chatelin, Spectral Approximation of Linear Operators, no. 65 in Classics Appl. Math., SIAM, Philadelphia, 2011, https://doi.org/10.1137/1.9781611970678.

[23] C. Chicone and Y. Latushkin, Evolution Semigroups in Dynamical Systems and Differential Equations, no. 70 in Math. Surveys Monogr., American Mathematical Society, Providence, 1999.

[24] R. F. Curtain and H. Zwart, An Introduction to Infinite-Dimensional Linear Systems Theory, no. 21 in Texts in Applied Mathematics, Springer-Verlag, New York, 1995, https://doi.org/10.1007/978-1-4612-4224-6.

[25] O. Diekmann, P. Getto, and M. Gyllenberg, Stability and bifurcation analysis of Volterra functional equations in the light of suns and stars, SIAM J. Math. Anal., 39 (2008), pp. 1023-1069, https://doi.org/10.1137/060659211.

[26] O. Diekmann, P. Getto, and Y. Nakata, On the characteristic equation $\lambda=\alpha_{1}+\left(\alpha_{2}+\right.$ $\left.\alpha_{3} \lambda\right) e^{-\lambda}$ and its use in the context of a cell population, J. Math. Biol., 72 (2015), pp. 877908, https://doi.org/10.1007/s00285-015-0918-8.

[27] O. Diekmann and M. Gyllenberg, Equations with infinite delay: Blending the abstract and the concrete, J. Differential Equations, 252 (2012), pp. 819-851, https://doi.org/10.1016/ j.jde.2011.09.038.

[28] O. Diekmann, M. Gyllenberg, H. Huang, M. Kirkilionis, J. A. J. Metz, and H. R. 
Thieme, On the formulation and analysis of general deterministic structured population modelsii. nonlinear theory, J. Math. Biol., 43 (2001), pp. 157-189, https://doi.org/10.1007/ s002850170002.

[29] O. Diekmann, M. Gyllenberg, J. A. J. Metz, S. Nakaoka, And A. M. De Roos, Daphnia revisited: local stability and bifurcation theory for physiologically structured population models explained by way of an example, J. Math. Biol., 61 (2010), pp. 277-318, https: //doi.org/10.1007/s00285-009-0299-y.

[30] O. Diekmann, M. Gyllenberg, J. A. J. Metz, and H. R. Thieme, On the formulation and analysis of general deterministic structured population models I. Linear theory, J. Math. Biol., 36 (1998), pp. 349-388, https://doi.org/10.1007/s002850050104.

[31] O. Diekmann, S. A. van Gils, S. M. Verduyn Lunel, And H.-O. Walther, Delay Equations - Functional-, Complex- and Nonlinear Analysis, no. 110 in Appl. Math. Sci., SpringerVerlag, New York, 1995, https://doi.org/10.1007/978-1-4612-4206-2.

[32] K. Engelborghs, T. Luzyanina, K. J. In 'T Hout, And D. Roose, Collocation methods for the computation of periodic solutions of delay differential equations, SIAM J. Sci. Comput., 22 (2001), pp. 1593-1609, https://doi.org/10.1137/S1064827599363381.

[33] K. Engelborghs, T. Luzyanina, And D. Roose, Numerical bifurcation analysis of delay differential equations using DDE-BIFTOOL, ACM Trans. Math. Softw., 28 (2002), pp. 121, https://doi.org/10.1145/513001.513002.

[34] K. Engelborghs And D. Roose, On stability of LMS methods and characteristic roots of delay differential equations, SIAM J. Numer. Anal., 40 (2002), pp. 629-650, https://doi.org/10. $1137 /$ S003614290037472X.

[35] P. ERdös AND P. TURÁN, On interpolation I. Quadrature- and mean-convergence in the Lagrange-interpolation, Ann. Math., 38 (1937), pp. 142-155, https://doi.org/10.2307/ 1968516.

[36] W. FELler, On the integral equation of renewal theory, Ann. Math. Statist., 12 (1941), pp. 243267, https://doi.org/10.1214/aoms/1177731708.

[37] E. Fridman, Introduction to Time-Delay Systems: Analysis and Control, Systems Control Found. Appl., Birkhäuser, Basel, 2014, https://doi.org/10.1007/978-3-319-09393-2.

[38] G. Gripenberg, S.-O. Londen, and O. Staffans, Volterra Integral and Functional Equations, no. 34 in Encyclopedia Math. Appl., Cambridge University Press, Cambridge, 1990.

[39] K. Gu, V. L. Kharitonov, And J. Chen, Stability of Time-Delay Systems, Control Eng., Birkhäuser, Basel, 2003, https://doi.org/10.1007/978-1-4612-0039-0.

[40] J. K. Hale And S. M. Verduyn Lunel, Introduction to Functional Differential Equations, no. 99 in Appl. Math. Sci., Springer-Verlag, New York, 1993, https://doi.org/10.1007/ 978-1-4612-4342-7.

[41] M. Iannelli, Mathematical Theory of Age-Structured Population Dynamics, Giardini Editori e Stampatori, Pisa, 1995.

[42] H. InABA, Age-Structured Population Dynamics in Demography and Epidemiology, SpringerVerlag, Singapore, 2017, https://doi.org/10.1007/978-981-10-0188-8.

[43] T. Insperger And G. StépÁn, Semi-Discretization for Time-Delay Systems, no. 178 in Appl. Math. Sci., Springer-Verlag, New York, 2011, https://doi.org/10.1007/978-1-4614-0335-7.

[44] E. JARLEBRING, The spectrum of delay-differential equations: numerical methods, stability and perturbation, PhD thesis, Inst. Comp. Math. TU Braunschweig, 2008.

[45] W. O. Kermack AND A. G. MCKendrick, A contribution to the mathematical theory of epidemics, P. Roy. Soc. Lond. A Mat., 115 (1927), pp. 700-721, https://doi.org/10.1098/ rspa.1927.0118.

[46] R. Kress, Linear Integral Equations, no. 82 in Appl. Math. Sci., Springer, 3 ed., 2014, https: //doi.org/10.1007/978-1-4614-9593-2.

[47] Y. Kuang, Delay Differential Equations: With Applications in Population Dynamics, no. 191 in Math. Sci. Eng., Academic Press, San Diego, 1993.

[48] A. J. LotkA, On an integral equation in population analysis, Ann. Math. Statist., 10 (1939), pp. 144-161, https://doi.org/10.1214/aoms/1177732213.

[49] T. Luzyanina And D. Roose, Periodic solutions of differential algebraic equations with time delays: computation and stability analysis, Int. J. Bifurcat. Chaos, 16 (2006), pp. 67-84, https://doi.org/10.1142/S0218127406014599.

[50] K. A. Lythgoe, L. Pellis, And C. Fraser, Is HIV short-sighted? Insights from a multistrain nested model, Evolution, 67 (2013), pp. 2769-2782, https://doi.org/10.1111/evo.12166.

[51] N. MacDonald, Biological Delay Systems: Linear Stability Theory, no. 8 in Cambridge Studies in Mathematical Biology, Cambridge University Press, Cambridge, 1989.

[52] The Dynamics of Physiologically Structured Populations, no. 68 in Lecture Notes in Biomathematics, Springer-Verlag, Berlin, Heidelberg, 1986, https://doi.org/10.1007/ 
978-3-662-13159-6.

[53] W. Michiels And S.-I. Niculescu, Stability, Control, and Computation of Time-Delay Systems: An Eigenvalue-Based Approach, second edition, no. 27 in Adv. Des. Control, SIAM, Philadelphia, 2014, https://doi.org/10.1137/1.9781611973631.

[54] J. Mikusiński and C. Ryll-Nardzewski, Sur le produit de composition, Studia Mathematica, 12 (1951), pp. 51-57.

[55] T. J. Rivlin, An Introduction to the Approximation of Functions, Blaisdell Publishing Company, Waltham, MA, 1969.

[56] H. L. Royden and P. M. Fitzpatrick, Real Analysis, Prentice Hall, 4th ed., 2010.

[57] J. Sieber, K. Engelborghs, T. Luzyanina, G. Samaey, and D. Roose, DDE-BIFTOOL manual: Bifurcation analysis of delay differential equations, ArXiv e-prints, (2014), https: //arxiv.org/abs/1406.7144.

[58] H. Smith, An Introduction to Delay Differential Equations with Applications to the Life Sciences, no. 57 in Texts Appl. Math., Springer-Verlag, New York, 2011, https://doi.org/10. 1007/978-1-4419-7646-8.

[59] G. StÉpÁn, Retarded Dynamical Systems: Stability and Characteristic Functions, no. 210 in Pitman Research Notes in Mathematics Series, Longman Scientific \& Technical, Harlow, 1989.

[60] L. N. Trefethen, Spectral Methods in MATLAB, Software Environ. Tools, SIAM, Philadelphia, 2000, https://doi.org/10.1137/1.9780898719598.

[61] G. F. WebB, Theory of Nonlinear Age-Dependent Population Dynamics, Chapman \& Hall Pure and Applied Mathematics, CRC Press, 1985. 